\title{
Measuring the "Weight" of Human in vivo Bio-Inertia by Legendary Galileo Falling Body Experiments on a Commercial 10m Diving Platform and Gravitationally Inversion of Newton's Three Laws of Motion into the Basic Laws of Evolution
}

\author{
Yi Yu Lai \\ Innoen ${ }^{\circledR}$ Gravitational Institute, Buffalo, New York, USA \\ yylai[at]innoen.org
}

\begin{abstract}
Mutations of legendary Galileo falling bodies experiment with non-living objects in a non-isolated environment demonstrate that the recoverable internal motions of falling bodies can bind and polarize gravity over conventional Newtonian mass inertia. Bio quantum path experiments further interpret the binding mechanism and annotate the isolated logic restriction of Einstein's equivalence principle. Mutations of the Cavendish experiment reveal $10^{9}$ levels of gravitational differences between living and dead states. Mutations of the Galileo falling-body experiments for living beings confirmed that such differences come from recoverable internal motion surface tension gravitational binding that can be calibrated as a measurable bio-inertia. We then calibrated the falling height difference for human in vivo bio-inertia on a commercial 10m diving platform and verified $98 \%$ of populations on Earth can safely be tested in this way with enough preparation training. In vivo lifetime gravitational binding curve that governs all biological parameters and reveals life evolutionary mechanisms becomes technologically feasible. These results, along with various facts, modulate the gravitational multi-surface tension region resonating model of in vivo bio quantum path inversion superposition. Photoelectric effect, PCR, GPCR, ancient CSF-ligament human Kungfu training systems, music harmonics, and board observations physically sustain this model. Newtonian Third Laws of motion are therefore evolved into Basic Laws of Evolution originates from surface tension non-unitary time inversion superposition that is different from the mathematical superposition in quantum mechanics. The origin of memory \& think from surface tension region non-unitary inversion is also disciplinarily integrated.
\end{abstract}

Keyword: Bio-inertia, $F H D$, internal motion, gravitational binding, bio quantum path, inversion superposition

\section{Results, Empirical Section}

1.1 Mutations of legendary Galileo falling body experiment for non-living objects

Legend says that Galileo Galilei has dropped two cannonballs of different masses from the Leaning Tower of Pisa to show that their speed of descent was independent of their weight. One of the famous thought experiments acts as the physical basis of Newton's Three Laws of Motion. Now we mutate this experiment to understand the quiddity of this basis (We need to define the "falling height difference" indicator. Release two objects simultaneously from a certain height, at the moment when the first falling object hits on the ground, the height of the second object from the ground is defined as the "falling height difference". Legendary Galileo's experiment tells people for centuries that the "falling height difference" of the two objects he released from the Leaning Tower of Pisa is zero. Any significant non-zero "falling height difference" over the tolerable accuracy means the masses of two released objects are not independent of their weight. The Falling height difference is symbolized as $\boldsymbol{F H D}$, tested by camera video in this paper.):

\section{Mutation 1.1: Slinky free fall mutation}

(Fig.1a) shows two slinky toys with a free mounting height of $55 \mathrm{~mm}$ and a free suspension length of $1120 \pm 2 \mathrm{~mm}$. (For slinky toys, it is not easy to find two sets with the same free suspension length even if their mounting height is the same within measuring accuracy of $0.3 \mathrm{~mm}$ by a pair of Vernier calipers. We still carefully choose two sets with the same free mounting height and their free suspension length difference falls within $5 \mathrm{~mm}$ from hundreds of commercial products. The final minimum difference of the free suspension lengths of two selected slinky toys is $1118,1122 \mathrm{~mm}$; write as $1120 \pm 2 \mathrm{~mm}$.) We hang them as in (Fig.1b(1)), slinky A is free suspension, and slinky B is tied with a metal bar inside to prevent recoil back. Release them from the same height simultaneously, B hits on the ground first. Following the conventional Galileo experiment, the difference in the mass amount of the two rigid bodies will not influence the falling time; then, what makes the difference of the falling time should come from the difference in the inner motion of the non-rigid bodies. (After release, slinky A allows recoil back motions and the slinky $\mathrm{B}$ does not, the recoil back motion is a kind of inner motion that induces the differences of accelerations. In the experiment, a rack is used to calm the falling bodies to have a long enough stable time before releasing; thus, no extra holistic kinetic energy is resident on to the released objects. Such a non-external kinetic energy driving state shows maximum inner motion effects. The conventional Newtonian rigid body concept means inner motion is zero, the more inner motion, the more errors of the Newtonian system. Here just amplify the inner motions to show the hidden premises.)

Volume 10 Issue 9, September 2021 


\section{International Journal of Science and Research (IJSR) \\ ISSN: 2319-7064 \\ SJIF (2020): 7.803}

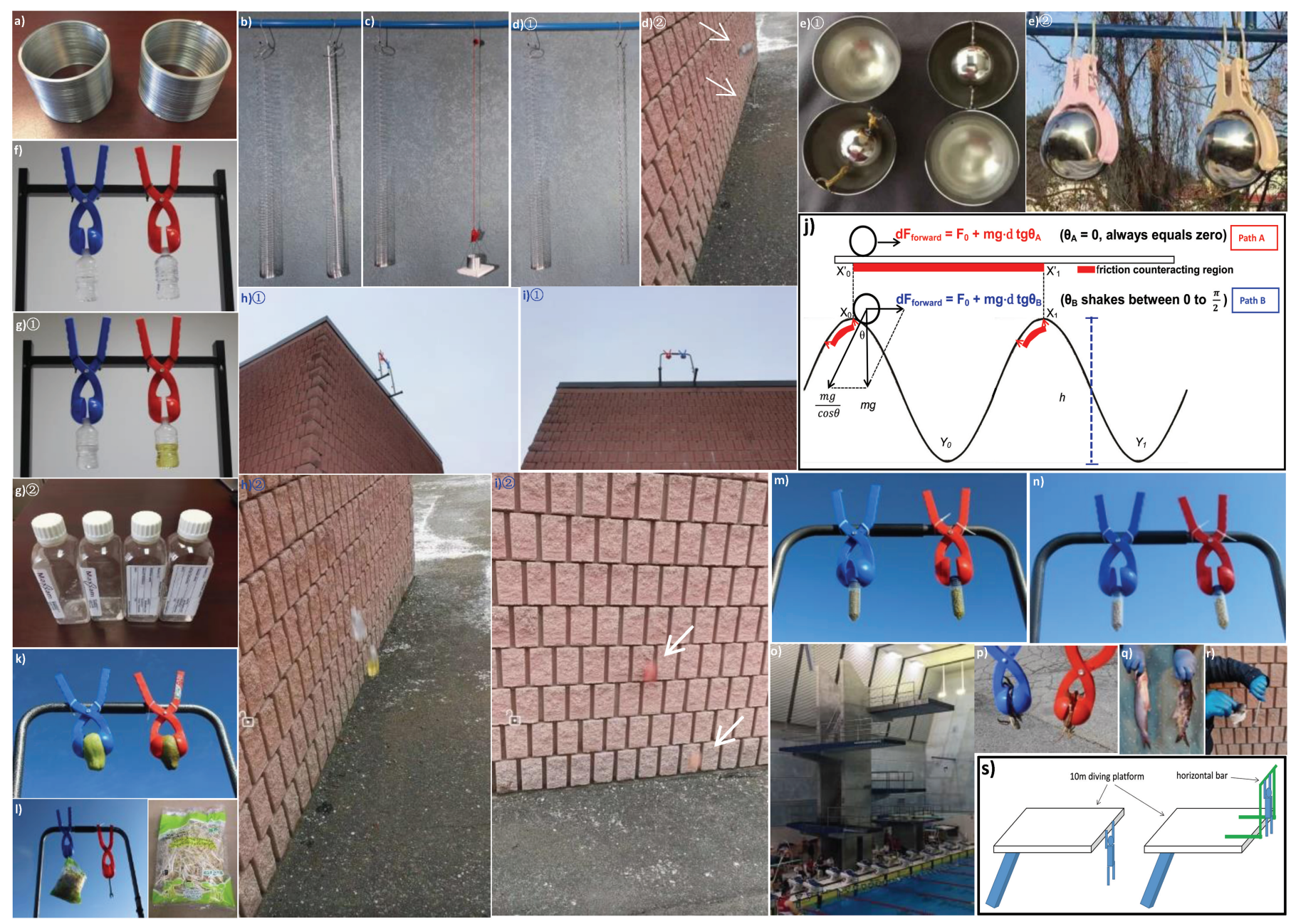

Figure 1: Measuring the "weight" of human in vivo gravitational waves by mutations of legendary Galileo falling body experiments

a) a pair of slinky toys with the same mounted height $\mathbf{b}$ ) hung up the slinky pair, A is free suspension, B with a metal rad fixed to avoid coil back c) A is free suspension, B mounted and tie with silk to avoid extension d) (1) A is free suspension slinky, B is a same length metal chain d) (2) the snapshot of the pair from d) (1) released from height (Suppl. Movie 3) e) (1) inside view of the bi-layer metal balls e) (2) hang up the bi-layer metal balls for experiment $\mathbf{f}$ ) a bottle pair hung on the rack, one with half bottle of water and another with full water, note the ice-cream clips design by a six-year-old girl $\mathbf{g}$ ) (1) a bottle pair hung on the rack, A with half bottle of water, B with half bottle of oil $\mathbf{g}$ (2) the 4 laboratory bottles among more than one thousand commercial bottles for mutation $1.5 \mathbf{~ h}$ ) (1) the bottle pair from g) (1) hang on the rack from height for experiment h) (2) the snapshot of the bottle pair experiment, refer to (Suppl. Movie 4) i) (1) apple pair hang on the rack from height, one is a fresh apple and another is cooked apple i)(2) snapshot of the apple pair from i) (1), refer to (Suppl. Movie 6) j) bio quantum path, refer to (Suppl. Movie 5) k) chayote fruit pair hang on the rack, one is fresh, another is cooked l) a commercially packed vegetable bag experiment with a metal weight, a solid metal ball is not obvious in photos, so we use the metal parts in this shape instead. The FHD between a cooked organism and the metal weight is quite small, therefore, while a sample is rare to acquire we then use the metal weight to replace the cooked sample $\mathbf{m}$ ) mung bean seeds hang on the rack, one is raw seeds and another is soaked for $24 \mathrm{H} \mathbf{n}$ ) soybean seeds hang on the rack, one is raw seeds and another is soaked for $24 \mathrm{H} \mathrm{o}$ ) the $10 \mathrm{~m}$ diving platform we performed the first human bio-inertia trial in the world in Etobicoke, Ontario, Canada at Feb. of 2020. p) crab free-fall experiment, one is alive, and another is cooked q) fish free-fall experiments, one is living, and another is cooked. Before the experiment, we carefully choose two fishes with the same length: however, the cooked one is shortened after boiling. Deprive of bio-inertia also induce a space-time deformity. For these types of animals, it is difficult to use the "ice-cream" clips, we have to hand-hold for stable and then release $\mathbf{r}$ ) rat free-fall experiment with the metal weight, still need the handhold $\mathbf{s}$ ) sketch of the ideal diving posture with a rack for testing FHDs

NASA has a video on its website to show Apollo astronauts performed the Galileo experiment on the Moon in 1971 with a hammer and a feather as shortened in (Suppl. Movie 1). If we carefully observe the video, we could find the feather has already lagged the hammer for a few centimeters, also inversion a few times in the falling. NASA wanted to show the difference of masses will not impact the FHD of objects on the Moon without atmosphere. They did fail to realize that besides the atmosphere factor, the inner motion also plays a role. The feather is softer than a hammer; it possesses much more inner motion in the falling process than that of the former. Even under an environment without atmosphere friction like on the Moon, we can see such an inner motion difference has already induced a visible difference in the NASA experiment at a lower height. (If the falling distance is enlarged to over $20 \mathrm{~m}$, we could expect a larger observable $F H D$ originates from the inner motion difference between them.) From this video, we can say that mutation 1 equivalent experiment had been performed by NASA "unintentionally" on the Moon half a century ago. Shortly, while China landing

Volume 10 Issue 9, September 2021 
humans on the Moon, we'll suggest the legendary Galileo experiment slinky mutations to be executed there; we can then clearly see how the inner motions of a falling object impact gravity.

\section{Mutation 1.2: Le Châtelier's effect of slinky conservative force binding}

Slinky mutation of the Galileo experiment can also use (Fig.1c), slinky A is free suspension, and slinky B tied with thin threads while mounted to prevent stretch if falling. We hold slinky A and slink B with their bottom at the same level at the height and then released simultaneously. The FHD is still non-zero, B hits on the ground first. We put the bottom of A and B as the falling level is to show Le Châtelier's effect of gravity. As in (Suppl. Movie 2), release a free suspension slinky from a height, there is almost no displacement of the bottom until the top of it can reach the slinky uncompressed position. This type of motion is Le Châtelier's effect of gravity since the top gets the highest elastic potential which stores gravitational potential, and the bottom gets the minimum. This elastic potential is held by the hand, while release, Le Châtelier's effect will happen in the direction of reducing this potential; therefore, the highest potential points first move to reach an uncompressed position, then the whole structure moves down with some oscillations. (The concept of the "center of mass" doesn't fit for a slinky since it is shifting.) From here, gravity follows Le Châtelier's effect on non-rigid bodies in a free-fall state. In a surface tension region structure such as a soap bubble with difform motions, or a multi-surface tension bio-system, gravity can be shifted to various directions. Even under such complex conditions, the movements of gravity polarization regions still follow Le Châtelier's effect. (Gravity polarization roughly means change the direction of the gravity vector from its original or opposite direction to others induced by surface tension gravitational binding.)

\section{Mutation 1.3: modulation of internal motion from a slinky and a free chain}

We still have not differed between inner motion \& internal motion, now we'll show this conceptual difference. A is a free suspension slinky, and B is a metal chain with the same free suspension length as it (Fig.1d(1)), release them from height, the metal chain will hit the ground first as in (Fig.1d(2)) and (Suppl. Movie 3). Both the slinky and the chain are not rigid bodies; the only difference is that the motion of the slinky is highly recoverable and the metal chain is almost no recoverable. Take Hamiltonian, $\widehat{H}=\widehat{T}+\widehat{V}$ (T: kinetic energies, $\mathrm{V}$ : potential energies), the more gravitational potential energy is transferred to total energy, the more internal motion an object will possess. For the metal chain, the transferring efficiency from the gravitational potential is lower, and the efficiency of maintaining the transferred gravitational potential energy by internal motion is also lower than that of the elastic slinky. The Hamiltonian can be written as $\widehat{H}_{\text {Internal }}=\widehat{V}_{\text {gravity }}$ since the system is not sensitive to $\widehat{T}$. (In bio-systems, almost all motions are internal motions; it is the reason why it is challenging to transfer kinetic energy to an animal. For a non-living rigid metal ball, we can take another metal ball to hit it, follows the Law of Conservation of Momentum, $p=\int m_{i} v_{i}$, total kinetic energy is easily transferred to it and show out. For an animal, it is difficult to increase its system kinetic energy to it by hitting a metal ball on it due to its $\widehat{H}_{\text {Internal. }}$. If we use this way, the holistic kinetic energy of the metal ball needs to first transfer to an in vivo potential energy type to the whole body of the animal, then follow the potential energy structure regenerate back into kinetic energy. The recovering efficiency of kinetic energy in this process is quite lower. From this fact we then can know that the classical physical Law of Conservation of Momentum and the Conservation of Energy is also not fit for bio-systems due to the high percentage of in vivo internal motions maintained and the potential energy structure involved. The ancient physical strike training method $(\mathrm{p} 1681, \rightarrow)^{1}$ was designed for improving the internal motion gravitational binding of human bodies, with more than 1500 years of history. The mechanism is still the human body can transfer more kinetic energy to potential energy after training. The process also does not fit the law of the conservative of momentum since this law only experimented with rigid bodies. Now, we can differ the concept of internal motion VS inner motion; for a non-rigid body, internal motion generally means recoverable motion and inner motion does not. We can easily understand that the conservation of momentum relies on rigid body characteristics (Later we'll see, the transferring of internal motion must rely on surface tension). The original format of Newton's Second Law of motion is the impulse-momentum theorem: $\vec{F}=k \cdot \frac{d(m \cdot \vec{v})}{d t}$ ), even rely on rigid body conditions more than the later version, $\vec{F}=m \vec{a}$.

\section{Mutation 1.4: two bi-layer metal balls free-fall experiment to modulate relative mobility}

As in (Fig.1e(1), we order 8 stainless steel hemispheres, four with $100 \mathrm{~mm}$ radii, and four with $51 \mathrm{~mm}$ radii, thickness $1 \mathrm{~mm}$, from a manufactory with ISO standards. Four small hemispheres were soldered into two $51 \mathrm{~mm}$ metal hollow balls, one hollow ball also soldered with two small rings and fixed into one larger hemisphere with two rubber or metal springs. After the small $51 \mathrm{~mm}$ hollow ball was fixed into one larger hemisphere by rubber bands or metal springs, solder another hemisphere on it (assure not damage the rubber bands). Then we get a $100 \mathrm{~mm}$ hollow ball with rubber bands or metal springs to suspend a $51 \mathrm{~mm}$ hollow metal ball inside it. Another small hollow ball was soldered inside another hollow hemisphere with two metal rods. Finally, we get two bi-layer hollow metal balls, A with inner small metal hollow ball connects to the larger metal ball with rubber bands or metal springs. The B is similar to A; just the connection is soldered metal bars, then it is difficult to swing compares to that of A. Release them from a height as in (Fig.1e(2)), B hits on the ground first (with 5 min of calm time on the rack). This mutation can modulate the internal motion clearer; in this case, the small hollow metal ball relatively oscillates to the large metal ball or the whole system. We can call this type of internal motion as relative mobility which adapts to in vivo conditions well; it can bind gravity. (This mutation has not only modulated relative mobility but also excluded air friction. For slinky mutation 1.1, some picky people possibly argue with the difference air friction of the A, B treatment; now, the air frictions of the two hollow metal balls are the same. Such arguments are then excluded by the two $100 \mathrm{~mm}$ bi-layer hollow metal balls with different inner metal ball 
layer's relative mobility under the strictest empirical conditions.)

\section{Mutation 1.5: free fall of bottles with different mobile liquid contents}

(Fig.1f) shows two commercial water bottles, the B full of water, no visible bubbles, A only with half bottle of water, release them from height, B falls on the ground first. We should carefully note that the reason that $B$ hits first is not due to that $\mathrm{B}$ is heavier than $\mathrm{A}$. That is due to the water inside $\mathrm{A}$ (half bottle) possesses higher relative mobility to the bottle wall than that of B (which fully occupies the bottle, then the water relative mobility is confined). From the next experiment, we can see the impact of relative mobility on FHD more clearly. (Fig.1g(1)) shows two commercial water bottles, A with a half-bottle of water and $\mathrm{B}$ with a half-bottle of plant oil, release them from height, B hits on the ground first. While design these two experiments, I thought they need a very high place to show a visual $F H D$, even prepare to rent a helicopter. However, only with $4.82 \mathrm{~m}$ height, they did show an indisputable $F H D$ (Fig.1h(1), Fig.1h(2)). (Suppl. Movie 4) shows a video with the original video binds with its slow-motion version together; the bottles come from two $330 \mathrm{ml}$ drinking water bottles in the same batch. We can see from (Fig.1h(2) snapshot, the FHD between half a bottle of vegetable oil and half a bottle of water has already attained around 1.5 bottle length from a height of $7.04 \mathrm{~m}$, which is, $1.5 \times 14.5=$ to $21.75 \mathrm{~cm}$. (The length of the bottles in the video is $14.5 \mathrm{~cm}$, diameter $6.5 \mathrm{~cm}$. Before releasing the bottles, we need to hang them on a rack in still states for at least $5 \mathrm{~min}$; it is to assure two same height bottles do not get extra holistic kinetic energy, or the difference of the total energy of two released objects compare to their height-related potential energies is minimized. The human hands cannot stably hold before releasing objects for a long time; therefore, a rack (Fig. 1f, etc.) is necessary to assure the above least difference in all the nonliving being mutations. The releasing process needs two persons to open the clips simultaneously.) This mutation has been performed in Guizhou, Shanxi, and Heibei Province of China; Ontario of Canada; Buffalo, New York, USA, for around 600 experiments at different places, from different falling heights from $4.82 \mathrm{~m}$ to $23.65 \mathrm{~m}$, with different types of commercially available drinking bottles from $330 \mathrm{ml}, 500 \mathrm{ml}$, and $750 \mathrm{ml}$ (in all the 600 experiments, only four laboratory containers were used, as in (Fig.1g(2)), the remaining 1200 more bottles were commercial drink water bottles), different oil types from canola oil, vegetable oil, sunflower oil, peanut oil, soybean oil, car engine oil. The FHD difference is unavoidably presented in every trial (It is recommended that the oil can't contain any semi-solid clots.); in contrast, no single falling experiment from all above 600 trials could get any two bottles with $F H D$ result lower than $20 \mathrm{~cm}$, and the oil bottle unexceptionally hit first. We carefully arranged so many tests just to assure that longitudes, latitudes, altitudes above sea level, types of oils, locations, etc., will not impact the relative mobility driving result. Also, to reliably assure the resulted models have been totally disregarded by all the extant physical theories. We use commercial products instead of laboratory containers to perform the experiments, just to give the data reproducibility to the global public then every unprofessional people can validate the results directly. (This experiment is so simple that a six-year-old kindergarten girl has participated in the video of (Suppl. Movie 4, 6), with rock climbing safety rope on the waist. This six-year-old kindergarten girl not only performed experiments to challenge Newtonian, Einstein, Planck, and Schrödinger but also design a device to defeat me. In the beginning, I designed mechanical devices to hold slinky for a long time before releasing them. Without such devices, a slinky will shake to damage the result. It was the kindergarten girl whose favorite ice cream told me to use the clips. Her ice-cream clip "devices" (Fig.1f) did perform better than all of my designs.) For the concept of "hitting the ground simultaneously", the smallest bottle diameter is $6.5 \mathrm{~cm}$, length $14.5 \mathrm{~cm}$. Generally, human eyes can discern an $F H D$ of $0.5 \mathrm{~cm}$, even if we enlarge the condition "hitting the ground simultaneously" from $0.5 \mathrm{~cm}$ to a wide 6.5 $\mathrm{cm}$ tolerated range, the ideal story which stated in the legendary Galileo falling body experiment still never existed. The Leaning Tower of Pisa is 58.36 meters tall, the largest height of our 600 trials only $23.65 \mathrm{~m}$ which failed to attain the half-height of that Tower, and we also enlarged the tolerable measuring range, even so, no single piece of result can get a zero $F H D$ within acceptable standards. If half a bottle of water and half a bottle of oil are released from that famous Tower, we can expect a much larger $F H D$ than those in all our validations. (This fact means that all over the world, people have lived in the illusion of a legendary system for centuries. It is not the mistakes of Galileo or Newton, for rigid bodies, they were not wrong, and they had transcended their era greatly. However, the problem is the whole human society believes the isolated logic methods that make Galileo or Newton's success is the only approach for scientific study for centuries. Such an illusion does restrict the regenerative of science. Their methods are one of the successful approaches, but not inclusive of all! People who published the legendary Galileo experiment in textbooks revealed that they "expected" the legendary results instead of really "observing" the experiment.)

Before releasing, the oil bottle measured $173.274 \mathrm{~g}$, and the water bottle measured $184.125 \mathrm{~g}$ in the video. The lighter object falls on the ground first, which means relative motility has become the critical impact factor in FHD than those of the weights or the masses. We only weighed the two bottles for preparing the video, not for all the 600 trial experiments. For them, just poured half a bottle of water and half a bottle of oil; visually checked that they were at the same height. However, due to the density difference, the oil bottles should be lighter than those of the water bottles by visual check for over $98 \%$ of the conditions. And all of them got the result that the oil bottle hit the ground first. From here, we can see that relative mobility has exceeded mass or weight in the $F H D$ for non-rigid bodies. This reveals there exists "incompleteness" for Newton's and Einstein's systems for explaining weight, mass, and gravity under high potential energy bodies (with more surface tension regions). For these types of objects, gravitational waves are observable oscillation energy that is much higher in quantity than those acquired from rigid body models, and mass concepts will shift.

Mutation 1.6: liquid nitrogen mutation of bottle pairs (with a half-bottle of oil and a half bottle of water)

The quiddity of our mutation experiments refers to as the weak equivalence principle (WEP) in conventional physics. 
(Tab. 1) shows the historical validations of this principle in macrocosmic conditions (slightly modified from the original reference book ${ }^{2}$ and only keep the references of Newton and Eötvös. The original book has a reference index for each person in the table.); (Tab. 2) shows the modern validations in microcosmic terms.

Table 1: Historical test of weak equivalence principle (WEP) in the macrocosmic world ${ }^{1}$

\begin{tabular}{|c|c|c|c|}
\hline Year & Investigator & Sensitivity & Method \\
\hline $500 ?$ & John Philoponus & small & Drop tower \\
\hline 1585 & Simon Stevin & $5 \times 10^{-2}$ & Drop tower \\
\hline $1590 ?$ & Galileo Galilei & $2 \times 10^{-2}$ & $\begin{array}{l}\text { Pendulum, drop } \\
\text { tower }\end{array}$ \\
\hline 1686 & Isaac Newton $^{3}$ & $10^{-3}$ & Pendulum \\
\hline 1832 & Bessel & $2 \times 10^{-5}$ & Pendulum \\
\hline $\begin{array}{l}1908 \\
(1922)\end{array}$ & Loránd Eötvös ${ }^{4}$ & $2 \times 10^{-9}$ & Torsion balance \\
\hline 1910 & Southerns & $5 \times 10^{-6}$ & Pendulum \\
\hline 1918 & Zeeman & $3 \times 10^{-8}$ & Torsion balance \\
\hline 1923 & Potter & $3 \times 10^{-6}$ & Pendulum \\
\hline 1935 & Renner & $2 \times 10^{-9}$ & Torsion balance \\
\hline 1964 & Dicke, Roll, Krotkov & $3 \times 10^{-11}$ & Torsion balance \\
\hline 1972 & Braginsky, Panov & $10^{-12}$ & Torsion balance \\
\hline 1976 & Shapiro, et al. & $10^{-12}$ & Lunar laser ranging \\
\hline 1981 & Keiser, Faller & $4 \times 10^{-11}$ & Fluid support \\
\hline 1987 & Niebauer, et al. & $10^{-10}$ & Drop tower \\
\hline 1989 & Stubbs, et al. & $10^{-11}$ & Torsion balance \\
\hline 1990 & Adelberger, et al. & $10^{-12}$ & Torsion balance \\
\hline 1999 & Baessler, et al. & $5 \times 10^{-14}$ & Torsion balance \\
\hline 2017 & MICROSCOPE & $10^{-15}$ & Earth orbit \\
\hline
\end{tabular}

"?" in the table means "lack reliable direct historical documents, only indirect references available", or simply marked as "legendary".

Table 2: Validation of weak equivalence principle (WEP) in the microcosmic world

\begin{tabular}{|r|c|c|c|}
\hline Year & Investigator & Sensitivity & Method \\
\hline 2004 & Fray $^{5}$ et al. & $10^{-7}$ & Atomic Interferometer \\
\hline 2015 & Zhou $^{6}$ et al. & $10^{-8}$ & Atomic Interferometer \\
\hline 2017 & Rosi $^{7}$ et al. & $10^{-9}$ & Atomic Interferometer \\
\hline
\end{tabular}

The principle refers to the equivalence of gravitational and inertial mass in Einstein's general theory of relativity. Historically, the first free fall validation should perform by Simon Stevin at the Delft Church Tower in 1586 (He also contributed his role in 12-TET.). Not surprisingly, the legendary Galileo's story lacks narration from himself albeit it is so famous, only available a biography from his student: Vincenzo Viviani. As in the table, even as earlier as Newton's time, this experiment had reached a higher enough sensitivity. Later, accuracy was continuously enhanced. However, the claimed higher sensitivity of those validations in (Tab.1) totally relied on the fact that the solid metal of used objects closed to rigid bodies, thus restricted the internal motions (relative mobility of the different parts) of them in the experimental processes. Once experiments use non-rigid bodies that can show significant geometrical shifting in the falling processes, such as the mutation 1.5 of bottle contents, or mutation 1.4 of bi-layer metal balls, the accuracy will quickly be decayed to an unacceptable level. To confirm this non-rigid body impact hypothesis, we perform the nitrogen mutation of the mutation 1.5. As mentioned before, in more than 600 experiments, each with a half-bottle of oil and a half bottle of water fell from over $4.82 \mathrm{~m}$, all acquired FHDs > $20 \mathrm{~cm}$; that is due to the different relative mobility of the oil and the water inside the bottles. We then put 12 bottles into a liquid nitrogen jar, six $330 \mathrm{ml}$ and six $500 \mathrm{ml}$ commercial drinking water bottles, compose six experimental pairs (a half bottle of oil and a half bottle of water), and another 12 equally prepared bottles without nitrogen treatment as $\mathrm{CK}$, released from the heights of $5.28 \mathrm{~m}, 7.04 \mathrm{~m}$, and $23.65 \mathrm{~m}$; each pair hits on the ground simultaneously as in (Tab.3):

Table 3: Liquid nitrogen mutation for $F H D(s)$ of bottle pairs with a half-bottle of oil and a half bottle of water

\begin{tabular}{|c|c|c|c|}
\hline $\begin{array}{c}\text { falling } \\
\text { height }(\mathrm{m})\end{array}$ & $\begin{array}{c}\text { commercial } \\
\text { bottle size } \\
(\mathrm{ml})\end{array}$ & $\begin{array}{c}\text { liquid nitrogen } \\
\text { treated pair } \\
F H D(\mathrm{~cm})\end{array}$ & $\begin{array}{c}\text { CK group (without } \\
\text { liquid nitrogen) } \\
F H D(\mathrm{~cm})\end{array}$ \\
\hline \multirow{2}{*}{5.28} & 330 & 0 & 21 (water bottle first) \\
& 500 & 0 & 22 (water bottle first) \\
\hline \multirow{2}{*}{7.04} & 330 & 0 & 28 (water bottle first) \\
& 500 & 0 & 34 (water bottle first) \\
\hline \multirow{2}{*}{23.65} & 330 & 0 & 44 (water bottle first) \\
& 500 & $<2$ & 39 (water bottle first) \\
\hline
\end{tabular}

* non-detectable recorded as 0 . Liquid nitrogen frozen bottles only stable on the rack for 20s after hanging, lower than the general $5 \mathrm{~min}$ calm time for other mutation experiments. The height is measured by a laser distance meter that can reach $\mathrm{mm}$, the $F H D$ is estimated from a video which can touch $0.5 \mathrm{~cm}$ accuracy; both are rounded to $\mathrm{cm}$. This mutation was only performed in Ontario of Canada with 12 bottles to validate the relative rigid body hypothesis, and the CK equivalent experiments have been performed on different locations of Earth with enough generality.

From here, we can further see how internal motion (relative mobility) impact FHD. Historical experiments in (Tab. 1) used solid metal objects; the falling time was only seconds $(9.8 \mathrm{~m} / \mathrm{s})$. In so short a time, the geometrical shifting of those solid metal objects is totally undetectable; therefore, the $F H D$ s induced by the internal motions are also undetectable. While we change the solid metal into liquids inside bottles, the geometrical shifting of the liquids in the short falling time becomes obvious; therefore, it easily shows detectable FHDs. Now, we use liquid nitrogen overnight to fix these liquids again, then in the falling process, no detectable geometrical shifting or relative mobility for liquids inside liquid nitrogen frozen bottles, the results recover back to conventional undetectable $F H D$ again. Liquid nitrogen frozen bottles close to metal weights in historical experiments, this proves again that internal motions of an object play a critical role in the FHDs; we call such difference as internal motion Le Châtelier gravitational binding, which means inside the internal motion gravitational binding structures, Le Châtelier effect controls substantial parameters. (We also note, the term "rigid body" originated in classical physics should be a relative concept that concerns a specific process. Like a half bottle of liquid, if the geometrical parameters changed during the free-falling process; then, it is a non-rigid body. And while we froze it with liquid nitrogen, the geometrical parameters are also frozen in that falling process; thus, it is a rigid body that relates to the falling process. The highest falling distance of our experiments is only $23.65 \mathrm{~m}$; liquid nitrogen frozen bottles are rigid bodies that relate to free falling with this height. If the bottles fall from a helicopter with thousands of meters in height in which the liquid inside them will melt, then they are not the "rigid bodies" relate to that free fall process of that height. The "relative rigid body" concept or the "relative mobility" is vital for biological study because up to date, only relative mobility can completely calibrate bio-inertia. All other conventional biological indicators, such as DNA, protein structures, antibody titer, etc. are quite reluctant for

\section{Volume 10 Issue 9, September 2021}


this purpose. This is possibly the reason that senescence can't be modulated by these biological indicators.)

In classical physics, the corollary that "inertial mass equals to gravitational mass" is the foundation of Newtonian and Einstein's system, even classical quantum mechanics indirectly concern with it. Now from mutation 1 till mutation 6 of the legendary Galileo falling body experiment, we can clearly see that this foundation is only conditional true for rigid bodies. Once the internal motions (relative mobility) of objects reach certain levels, this foundation will be melting. Besides the legendary Galileo experiment, Eötvös experiment ${ }^{4}$ with $10^{-9}$ levels of accuracy is also referred to as important evidence of the abovementioned corollary. Now, if we change the solid metal rigid body masses in the Eötvös experiment to our bi-layer metal balls (Mutation 1.4), or bottles of liquids (mutation 1.5), we can see how the officially claimed $10^{-9}$ levels of accuracy will decaying. And due to entirely relying on the foundation, no existing theories in physics have ever experimented or theoretically clarified that internal motion or relative mobility of a system can bind gravity or conservative forces. Such kind of "physical disciplinary neglected" relative mobility gravitational binding is where life originating and evolving. ((Tab. 2) refers to the validation of WEP in the microcosmic world; at this level, these validations still seriously neglect the internal motion gravitational binding that originated from the surface tension region, which we will discuss later.)

\section{Bio quantum path experiment and the definition of bio-inertia}

The bio quantum path model has been initiated by the paper $(\text { p1682, } \rightarrow)^{1}$ with (Fig. 1j) and (Suppl. Movie 5). A ball running on path B gets the help of gravity, $d F_{\text {forward }}=F_{0}+$ $m g \cdot d \operatorname{tg} \theta \cdot \theta$ periodically change from 0 to close to $\frac{\pi}{2}$ thus it can "splice out" non-conservative forces such as frictions. On path A the ball only gets $d F_{\text {forward }}=F_{0}$ to overcome the friction, then the friction counteraction regions represented by the red color in the figure will be significantly larger than those on path $\mathrm{B}$; path $\mathrm{B}$ can then accumulate more gravitational to counteract frictions. This explanation still utilizes the Newtonian inertia; now, we'll see a totally different kind of inertia:

From (Fig. 1j), we can write the force that drives the ball on path $\mathrm{B}$ as, $d F_{\text {forward }}=F_{0}+m g \cdot d \operatorname{tg} \theta_{B}$, equally, the force that drives the ball on path A still can be written as, $d F_{\text {forward }}=F_{O}$ $+m g \cdot d \operatorname{tg} \theta_{A}$; the only difference between them is that $\operatorname{dtg} \theta_{B}$ always changes, and $d \operatorname{tg} \theta_{A}$ always equals to 0 , integral, then:

$$
\vec{F}=m \cdot \vec{a}+m \vec{g} \int \operatorname{tg} \theta \cdot d \theta
$$

Eq. 1 can be regarded as a modified Newton's second law of motion for non-rigid bodies. (The non-rigid body item $m \vec{g} \int \operatorname{tg} \theta \cdot d \theta$ can also be roughly called a "gravitational binding" item. We can easily find the law of conservation of momentum only effect for the rigid body item and the error of which will accumulate with the increasing of the non-rigid body gravitational binding item: $m \vec{g} \int \operatorname{tg} \theta \cdot d \theta$.) The physical significance of Eq. 1 means the persistent recovery rate of a physical parameter can bind extra gravity other than those written down in the rigid body format Newtonian laws. Here the angle $\theta$ between the moving vector and gravity is persistently shifting, therefore, it binds gravity. Suppose we choose other physical parameters, such as the metal ball height, speed, etc., still can get similar results; we call such a process as "parameter-against-gravity-recovery" gravitational binding movement. (We can also understand such a "parameter-against-gravity-recovery" process by Polymerase chain reaction (PCR). People put PCR premix including a DNA template, polymerase, two DNA primers, dNTPs, buffer solutions, etc., into a PCR tube; then use thermal cycles (15-40 cycles, each cycle: denaturation: 94-97, annealing: 50-65, elongation: $70-75^{\circ} \mathrm{C}$ ) to synthesis DNA segments for analysis. Each in vitro thermal cycle is a temperature parameter-against-gravity-recovery process; therefore, accumulates gravitational binding or bio-inertia for the DNA synthesis requirements. In vivo processes are similarly need "parameter- against-gravity-recovery" to bind gravity for life function, just the binding capacity enlarged greatly by all parameters get well optimized thus not like the in vitro PCR process needs so drastic temperature parameter cycles. The basic biological function for such "parameter-against-gravity -recovery" bio-inertia is to maintain the system parameter pairs by inversion. Suppose a research target can be described by bundles of parameters and each one with a certain minimum \& maximum value. For non-living systems, there are no correlations among these minimum $\&$ maximum value pairs, or the parameter pairs are ephemeral. For bio-systems, all the minimum \& maximum value pairs are strongly correlated by inversion to maintain the system lifespan; the lifespan of a bio-systems means the period while such a correlation is effective, senesces is the decaying of such parameter pair "correlations". (The inversion means some parameter pairs fluctuated from minimum to maximum, then other pairs reversely fluctuate from maximum to minimum as compensation, then all the system parameter pairs try to maintain for a long time. Suppose an animal lives well in a temperature from $10-20^{\circ} \mathrm{C}$, due to environmental shifting, it is forced to survive under a temperature range of $0-10^{\circ} \mathrm{C}$. The adaptation capability from $10-20^{\circ} \mathrm{C}$ shift to $0-10^{\circ} \mathrm{C}$ is never like non-living systems can be attributed to certain isolable reasons such as cooling the system to adapt to a lower temperature. The bio-system adaptation capability originates from the inversion of other system parameters. Suppose the temperature fluctuation correlated to some parameters as protein $X_{\text {maximum }}^{\text {minimum }}$, gene $Y_{\text {maximum }}^{\text {minmum }}, R N A Z_{\text {maximum }}^{\text {minimum }}$, then to acquire the temperature adaptation range, the relevant protein $\mathrm{X}$, gene $\mathrm{Y}$, and RNA Z, all need to inverse from their previous states. It is the inversion from other parameter pairs that supply this capability, not certain isolable factors can issue this temperature adaptation. All these parameter pair "correlations" are operating by gravitational binding bio-inertia to a gradient, such as protein $X_{\text {maximum }}^{\text {minimum }}$ has five levels, gene $Y_{\text {maximum }}^{\text {minmum }}$ have 7 levels, etc., the spinal cord is the highest gradient that governs all bio-system parameter pair gradients. No matter how many parameter pairs are inside and how complex a bio-system is, all system parameters are working in this way. Lower evolved species lack the spinal cord, still possess similar mechanism structures. For our previous mutations 1.5 , half bottle of liquids, in the free-fall process, the liquids still oscillate the "parameter-againstgravity-recovery" process, due to oil or water possesses different interaction with the bottle walls or with different gravitational binding efficiency, then accumulates different FHDs. We must note, a bottle wall is critical, which acts as the

\section{Volume 10 Issue 9, September 2021}


wave function inversion protection we'll refer to later. We also note, inside different bottles, oil and water show different gravitational binding as in our experiments. If we mix water and oil inside a bottle and let them free fall, the gravitational binding of water and that of oil still can't get any optimization. However, if we blend the oil and water vigorously to get emulsion with small enough liquid particle sizes into a bottle, then the gravitational binding in the free fall can be transferred among oil particle complex and water particle complex since the surface tensions of liquid particle complex have been enlarged exponentially. For bio-systems, not to mention two types of liquids, the gravitational binding of thousands of in vivo liquids and other gel structures can be optimized to inversion quite well, the capability of such optimization still originates from the huge surface tension of bio-systems. We should realize that the surface tension forces are critical for gravitational binding inversion biological processes. The surface tension induced inversion is more general in the natural environment than all the isolated logic equations we have acquired since Newtonian time.)

Einstein's Theory of Special Relativity equation: $m=\frac{m_{0}}{\sqrt{1-\frac{v^{2}}{c^{2}}}}$, could be regarded as a special type of internal motion effect, the mass measurement of an object shifts with the speed means it binds more gravity with the speed parameter. However, the value from Einstein's equation is so small in quantity due to his "loyalty" to the Newtonian system to create another rigid body system without considering the role of surface tension in transferring gravitational waves. He never imagined that the Newtonian system hid large discrepancies even under nonrelativistic conditions while surface tension effects are large enough. From (Fig. 1j), the gravitational binding of the ball on path $\mathrm{B}$ should be equal to: $\overrightarrow{m g} \cdot t$, here $t$ is the time advance which the ball acquires on path $B$. If we use the previous experiments such as mutation 1.5, the gravitational binding of the water bottle should be larger than the oil bottle by: $\overrightarrow{m g} \cdot F H D$, here $F H D$ represents the failing height difference between the two bottles; so "huge" a visually watchable gravitational binding shift can't be acquired from any extant physical theories including Einstein's curved space equation. Now, we can simply define item $m \vec{g} \int \operatorname{tg} \theta \cdot d \theta$ or its superposition as bio-inertia. (In bio-systems, the vector of gravity usually shifts which is different from non-living beings always plumb to the ground; however, no matter the main vector or the integrated gravity change to any direction, bio-inertia still can be modulated with the recovery rate of any leading parameter VS gravity.) And "bio quantum path" concept can be regarded as the physical or mathematical function which reveals the gravitational binding accumulating by recoverable internal motions. No matter which way it is written down, the recovering shifting of parameters-against-gravity is the fundamental mechanism. In Einstein's theory, mass turns out to be part of a more general quantity called the energymomentum tensor. With the equivalence principle, this tensor is readily generalized to curved spacetime. Einstein's gravity is a pure geometric gravity since all other parameters can be logically equation from geometry. For biosystems, not just geometrical indicators, any parameters will issue Le Châtelier gravitational binding via internal motion difference if the concerning parameter pair is sensitive for this in vivo environment. Different from Einstein's system which other non-geometrical parameters can be logically acquired from the geometry, bio-systems geometry only have inversion relationships with other non-geometrical parameters. The gravitational binding via surface tension region will be larger than those acquired from rigid mass or a curved space.)

\section{Mutations of legendary Galileo falling body experiment for living beings}

The Cavendish experiment, performed in 1797-1798 by English physicist Henry Cavendish, was the first experiment to measure the gravitational constant $\mathrm{G}$ in the laboratory. Directly test the affinity of two living organisms on a Cavendish device is quite challenging. Mutations of the Cavendish experiment alternatively test the gravitational difference between the dead and alive state of living beings. The mutation experiments had been initiated in Tsinghua University, Beijing in 2012, with results show that the gravitational loss of a living being reached $0.14-3.8 \%$ (1684, $\rightarrow)^{1}$, which means the gravity has been enlarged for $10^{9}$ in bio-systems than those measured in historical Cavendish experiments for non-living beings. It is due to this level of enlargement; mutation of Cavendish experiments can be performed by the public instead of restricted to professional physicists with advanced skills. (The term "gravitational binding energy" has been used to designate the minimum energy which must be added to it for the system to cease being in a gravitationally bound state, with a formula: $U=\frac{3 G M^{2}}{5 R}$. This kind of energy is totally different from those in bio-systems induced by internal motions. To avoid confusion, the "gravitational binding" term in this paper refers to in vivo instead of in vitro conditions. In vivo means surface tension effects are large enough.)

It took years for the mutation of Cavendish experiments to be performed on different locations on Earth with various samples. However, such a 7-year probing fell into an imperfect experimental method since it cannot get in vivo gravitational binding in this way. Till Jan.8, 2020, we began to systematically use the falling body experiment for in vivo gravitational binding. (Before that time point, we only occasionally used the free-fall method to validate the Cavendish mutation experiments and did fail to realize it has surpassed the Cavendish mutation experiments for modulation bio-inertia.) (Suppl. Movie 6) shows an apple and a cooked apple fall from 7.04m; from (Fig.1i(2) snapshot we can get the FHD $(19 \mathrm{~cm}$ brick thickness, mortar bed $1.3 \mathrm{~cm}$ thickness): $2.7 \times 19+2 \times 1.3=53.9 \mathrm{~cm}$. (Suppl. Movie 7), (Suppl. Movie 8) are also part of the experiments in (Tab.4):

Table 4: The $F H D(s)$ of some plant and animal samples for their alive and dead state (autoclave well-cooked and then wipe clean water) from the legendary Galileo falling body experiment

\begin{tabular}{|c|c|c|c|c|c|c|}
\hline $\begin{array}{c}\text { Falling } \\
\text { Height }(\mathrm{m})\end{array}$ & $\begin{array}{c}\text { living spl VS cooked spl or a } \\
\text { metal weight* }\end{array}$ & $\begin{array}{c}\text { sample 1 } \\
F H D(\mathrm{~cm})\end{array}$ & $\begin{array}{c}\text { Sample 2 } \\
F H D(\mathrm{~cm})\end{array}$ & $\begin{array}{c}\text { Sample 3 } \\
F H D(\mathrm{~cm})\end{array}$ & Ave & STDEV \\
\hline 7.04 & cat fish & 37 & 25 & 31 & 31.00 & 6.00 \\
\hline
\end{tabular}

Volume 10 Issue 9, September 2021 
International Journal of Science and Research (IJSR)

ISSN: 2319-7064

SJIF (2020): 7.803

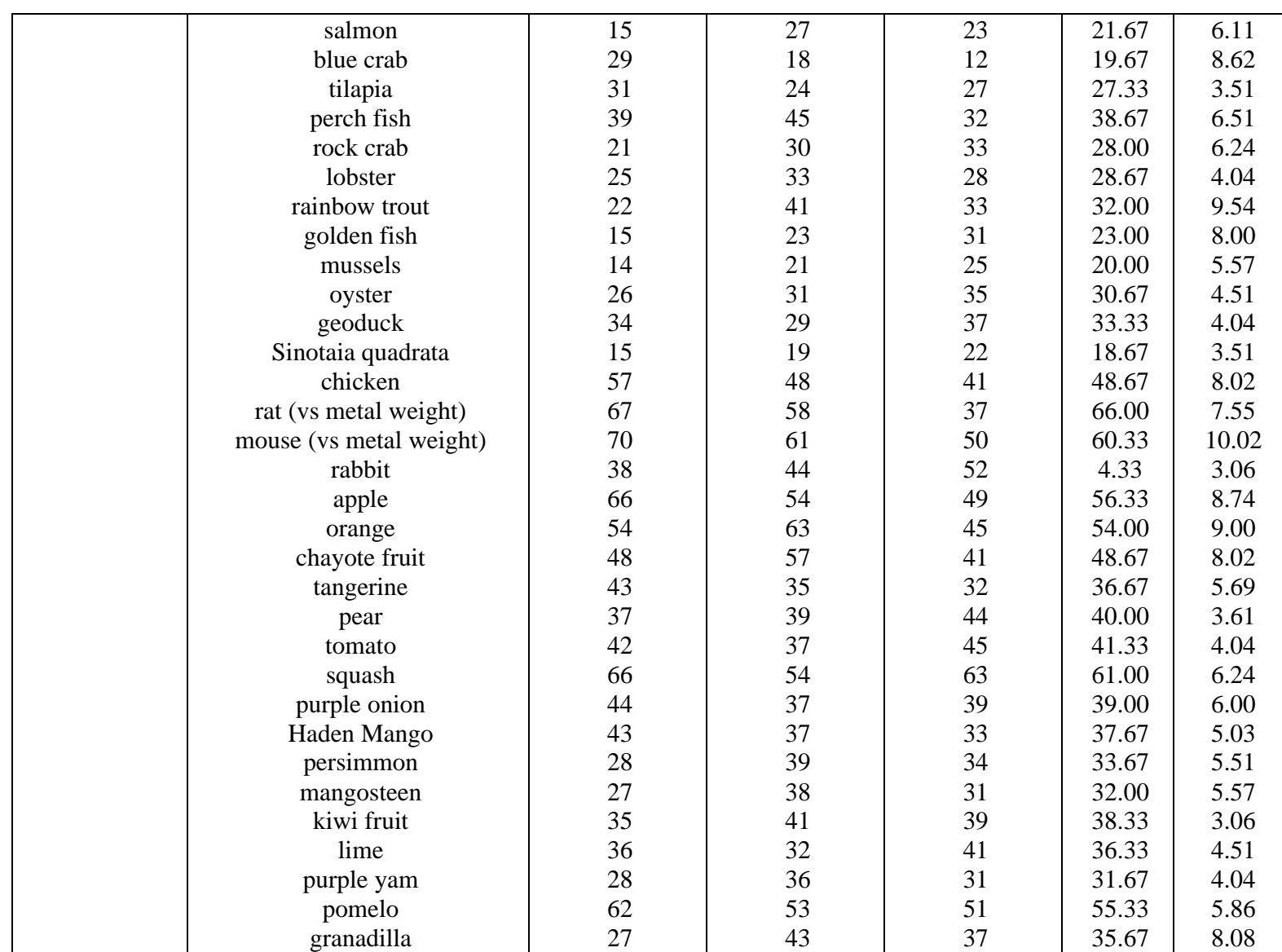

*** Most of the samples use the cooking method, while the sample is rare, use a solid metal weight (Fig.1r) to replace the dead sample. An autoclave is applied for the cooking method to assure cell death is well done. From fresh observation ${ }^{1}$, cell migration can be observed even after 9 hours of the killing of the animal. In the free-fall experiment, all samples need at least 30 seconds to settle time before releasing from the rack. Most of the samples are purchased from the markets without DNA identification to species since these tests are quite general to neglect species.

(Tab. 4) gives part of the FHD data of organisms with a falling height of 7.04 meters, which has been performed in China and Canada. From Newtonian mechanics:

$$
\begin{array}{rr}
\vec{F}=m_{a} \cdot \vec{a} & \vec{F}=G \frac{M_{\text {earth }} \cdot m_{G}}{r^{2}} \\
\text { Then } & m_{a}=\frac{g}{a} \cdot m_{G}
\end{array}
$$

For rigid bodies, Eq. 2 is a constant. For non-rigid bodies, it can be regarded as "holistic surface tension forces" or "inversion ratio". Due to different living beings possess diverse surface tension structures, $\mathrm{g} / \mathrm{a}$ is no longer a constant, which must test individually as in (Tab.4). For bio-systems, diverse parameters such as organism growth period, foods, mature stages, healthy conditions, etc. will play a role in the bio-inertia results. (Tab.5) uses two types of seeds to demonstrate the germination $F H D$ difference (Fig. 1m, mung bean; Fig. 1n, soybean.). Seed's germination activates metabolisms that are equivalent to internal motion; therefore, for all the samples released from $7.04 \mathrm{~m}$ height, raw seeds always hit the ground first. (If we regard Einstein's theory as a kind of gravitational binding by geometric indicators, bio-inertia still possesses "extra" binding from other parameters and the quantity levels are much higher than Einstein's gravity even if we only restrict it to geometric parameters. We can simply regard the mass inertia in Newtonian and Einstein's systems as "in vitro" inertia and internal motion gravitational binding as "in vivo" bio-inertia which can't be solely acquired from geometry.)

Table 5: Impact of seeds germination soaking to FHDs of soybean seeds and mung bean seeds by the legendary Galileo falling body experiment

\begin{tabular}{|c|c|c|c|c|c|}
\hline Seeds & $\begin{array}{c}\text { raw seeds weight }(\mathrm{g}) \\
\text { in the 50ml tube }\end{array}$ & $\begin{array}{c}F H D(\mathrm{~cm}), \text { Raw / } \\
24 \mathrm{H}, 3 \text { samples }\end{array}$ & Ave, STD & $\begin{array}{c}F H D(\mathrm{~cm}), \text { Raw / } \\
\text { 48H, 3samples }\end{array}$ & Ave, STD \\
\hline Mung bean & $46 \pm 0.27$ & $8,12,17$ & $12.33,4.51$ & $6,11,13$ & $10.00,3.61$ \\
Soybean & $40 \pm 1.03$ & $9,15,12$ & $12.00,3.00$ & $11,9,15$ & $11.67,3.06$ \\
\hline
\end{tabular}

*** For soybean and mung bean, select seeds with over $98 \%$ germination rate naturally put into a lab centrifuge tube to the $50 \mathrm{ml}$ marker and capped as $\mathrm{CK}, 24 \mathrm{H}$ $\& 48 \mathrm{H}$ means soaking the same seeds in water for $24 \mathrm{H}, 48 \mathrm{H}$ at $20^{\circ} \mathrm{C}$, use a paper towel to wipe clean the soaked seeds surface water and naturally put in the empty centrifuge tube to $50 \mathrm{ml}$ marker, cap; and measure the FHDs of raw seeds VS the soaked seeds, raw seeds never soaked to germination. However, in all $7.04 \mathrm{~m}$ releasing, always raw seeds hit the ground first. (Averagely from 10 tubes, raw seeds, $24 \mathrm{H}$ seeds, $48 \mathrm{H}$ seeds weigh $46 \pm 0.27,43 \pm 0.92,42 \pm 0.83$ grams for mung bean seeds, and 40 $\pm 1.03,37 \pm 1.26,36 \pm 1.14$ grams for soybean, respectively.) Here, we only perform two species, however, this experiment can adapt to all seeds or even animal embryos on Earth. Physiological activities sensitive to $F H D$ tests again validate the quiddity of life is gravitational binding or waves.

In (Tab.4), all the falling pairs composed of a living and dead organism show significant $F H D s$, to further confirm these
FHDs come from internal motion gravitational binding; we select some of the falling pairs to perform the liquid nitrogen

\section{Volume 10 Issue 9, September 2021 www.ijsr.net}


mutation again. We can see that the significant FHDs in (Tab.6) recover back to zero again, which means it is internal motion plays the role:

Table 6: Liquid nitrogen overnight makes the FHDs of living and dead falling organism pairs become zero.

\begin{tabular}{|c|c|c|}
\hline Falling Height $(\mathrm{m})$ & liquid nitrogen treated pair & $F H D(\mathrm{~cm})$ \\
\hline \multirow{5}{*}{5.48} & living apple - cooked apple & 0 \\
& living pear - cooked pear & 0 \\
& living tomato - cooked tomato & 0 \\
& living lobster - cooked lobster & 0 \\
& living mouse - metal weight & 0 \\
\hline \multirow{5}{*}{7.54} & living apple - cooked apple & 0 \\
& living pear - cooked pear & 0 \\
& living tomato - cooked tomato & 0 \\
& living lobster - cooked lobster & 0 \\
& living mouse - metal weight & 0 \\
\hline
\end{tabular}

* Pre-treatment same as in (Tab.4), then all experiment pairs are labeled and put into liquid nitrogen overnight. (Tab.4) supplies CK. (From these experiments, we can easily understand that a frozen process including liquid nitrogen storage does hurt life function.)

\section{Measuring of human in vivo gravitational binding on a commercial $10 \mathrm{~m}$ diving platform}

In 1907, Albert Einstein devised the "elevator" thought experiment, which was nothing less than the principles underlying the General Theory of Relativity. Einstein imagined an observer inside a closed elevator equipped with a complete physics lab, in which one can perform any physics experiment, but one cannot communicate directly with the outside world. He believed that no experiment performed inside the closed lab could distinguish between the lab's being in a strong gravitational field and its being accelerated rapidly upward. Einstein formulated this insight in what is known as the "equivalence principle". His curved space is theoretically composed of countless local "elevators". However, almost all our previous mutations can challenge such a rigid body insight. We can see, even if biological experiments are not allowed, non-living being mutations still compose the challenge. E.g., with the bi-layer metal ball pair or the half water bottle VS half oil bottle pair is released inside, if the enclosed lab is being in a strong gravitational field, then FHDs will be observed as demonstrated in our mutation experiments. However, if the pair is released in an enclosed lab that moving with acceleration g, then no $F H D$ can be presented since acceleration only contributes to the spatial displacement of the experimental pair members and cannot supply them internal motion gravitational binding which can accumulate the $F H D$ between them. From here, we can also understand that Einstein's gravitational lens that came from his elevator thought experiment is also not correct for in vivo or other conditions that surface tension is large enough. However, we should note, albeit Einstein's thought experiment is unsuccessful for general non-rigid bodies, the historical significance of it is still greatly pioneering. At least it does "unintentionally" remind us of the physical conditions of the bio-inertia that are fully different from the conventional Newtonian rigid body inertia as below:

1) bio-inertia is a type of internal motion binding process based on a strong gravitational field that it evolved from. If the strong gravitational field does not exist, only by an equivalent of acceleration, the binding process can't be fulfilled, or bio-inertia will not be accumulated to a detectable level. (We must note, the gravitational binding basis of a "strong gravitational field" can be further enhanced by bio-systems nearby. A person's gravitational binding is different while he is in an environment without living beings and in an environment with other bio-systems nearby. People prefer a biodiversity environment since in there they can get more gravitational binding.)

2) in bio-systems, gravitational mass never equals inertia mass due to the internal motion surface tension regions of bio-systems, or life automatically transfers the most kinetic energy into potential energy by a certain ratio. And the transferring process concerns environmental parameters, not belongs to any non-living being theoretical isolated models that can work under any environmental conditions. Once the transferring efficiency between these two types of energy is lower than a certain threshold means the life system is senescence or surface tension regions are damaged. (For dead bodies, the gravitational mass could roughly equal inertia mass since internal motions retrograded to difform movements which the gravitational binding efficiency similar to non-living objects.)

From the abovementioned Einstein's pioneering reminder, measuring bio-inertia becomes critical for theoretical understanding and application in bio-systems, especially the human in vivo bio-inertia. Unlike non-living beings that can test the object's weight or mass by a balance for calculating inertia from the Newtonian system, the only available in vivo measuring method for bio-systems is acquiring the FHDs since the bodyweight of an organism never concerns with its bio-inertia or health conditions. On the Feb.15 of 2020, we performed the first human in vivo bio-inertia measure in the world from the $10 \mathrm{~m}$ diving platform in Etobicoke, Ontario, Canada, with 8 volunteers as in (Tab.7).

Like the previous mutation experiments for non-living beings, we need to assure no extra system kinetic energy before diving. We first design the diving posture as in the left hand of the (Fig.1s), hope people can hold the brink of the platform for a while, and then release. However, this is impracticable even for athletes. We then realized that for the public test, it should make a horizontal bar rack like in the right hand of (Fig.1s), people hold the bar for enough stable time and release. This is the most ideal way for measuring bio-inertia. At that time, we lacked the horizontal bar rack as shown in the figure, then compromise to two postures as in (Suppl. Movie 9) and (Suppl. Movie 10) albeit they unavoidably have some errors than the ideal "horizontal bar rack release" posture. While people use these two inferior postures for diving, a metal weight as in (Fig. 1r) is releasing simultaneously to get the FHDs. (For the first posture in the video, people like a walk down from a step and naturally fall into the water. It looks simple however still needs some training to combat psychology. The second posture just naturally lies down backward, also needs some training to avoid extra body movements.) The diving water only prevents free-fall people from fall to death. We need to assure no extra holistic kinetic energy shift, therefore, diverse showy diving postures in 


\section{International Journal of Science and Research (IJSR) \\ ISSN: 2319-7064 \\ SJIF (2020): 7.803}

Olympic competitions are not allowed, all these showy styles will create wide errors for the $F H D$ results.

Table 7: Calibration of human in vivo bio-inertia by FHDs on a $10 \mathrm{~m}$ commercial diving platform

\begin{tabular}{|c|c|c|c|c|c|}
\hline volunteer & $\begin{array}{c}I^{\text {st }} \text { diving } \\
F H D(\mathrm{~cm})\end{array}$ & $\begin{array}{c}\text { 2nd diving } \\
F H D(\mathrm{~cm})\end{array}$ & $\begin{array}{c}3^{\text {rd }} \text { diving } \\
F H D(\mathrm{~cm})\end{array}$ & Ave & STDEV \\
\hline 1 & 41 & 56 & 67 & 54.7 & 13.05 \\
2 & 33 & 51 & 42 & 42.0 & 9.0 \\
3 & 46 & 29 & 37 & 37.3 & 8.50 \\
4 & 22 & 37 & 40 & 33.0 & 9.64 \\
5 & 75 & 66 & 82 & 74.3 & 8.02 \\
6 & 36 & 27 & 17 & 26.7 & 9.50 \\
7 & 45 & 58 & 33 & 45.3 & 12.50 \\
8 & 51 & 43 & 39 & 44.3 & 6.11 \\
\hline
\end{tabular}

*** For volunteers for Etobicoke diving club, they don't like to reveal their age and health condition, only follow two diving postures in the videos and train them to try to avoid extra body movements in the falling process. Everyone performed 5 diving with the same posture, the interval at least $20 \mathrm{~min}$, no eating or drinking between, record middle three FHDs to the table. We don't distinguish the age and gender; the names are also covered as requested by volunteers due to people nowadays still don't understand the method. We only got a few chances and suspended due to the COVID-19 pandemic. However, the significance of this test for future medical sciences and sportive applications is unprecedented. In a long period of history, no alternative way can replace the fundamental FHD test.

FHD results in the table will be influenced by various factors. Even for the same person to test at a different location, or with different styles of diving, etc., the results will be significantly different. However, if we can monitor the personal FHD standard curve on a lifetime basis with fixed test conditions, available life potential or bio-inertia will be unveiled. We have suggested the individual physical training and species gravitational binding pattern $(\mathrm{p} 1682, \rightarrow)^{1}$ before as in (Fig. 2a, 2b). To use the only available Cavendish mutation experiments in the past to get such curves is almost impossible; therefore, at that time these curves are only imaginary. Now, with the newly established FHD test, all similar curves become technologically feasible.
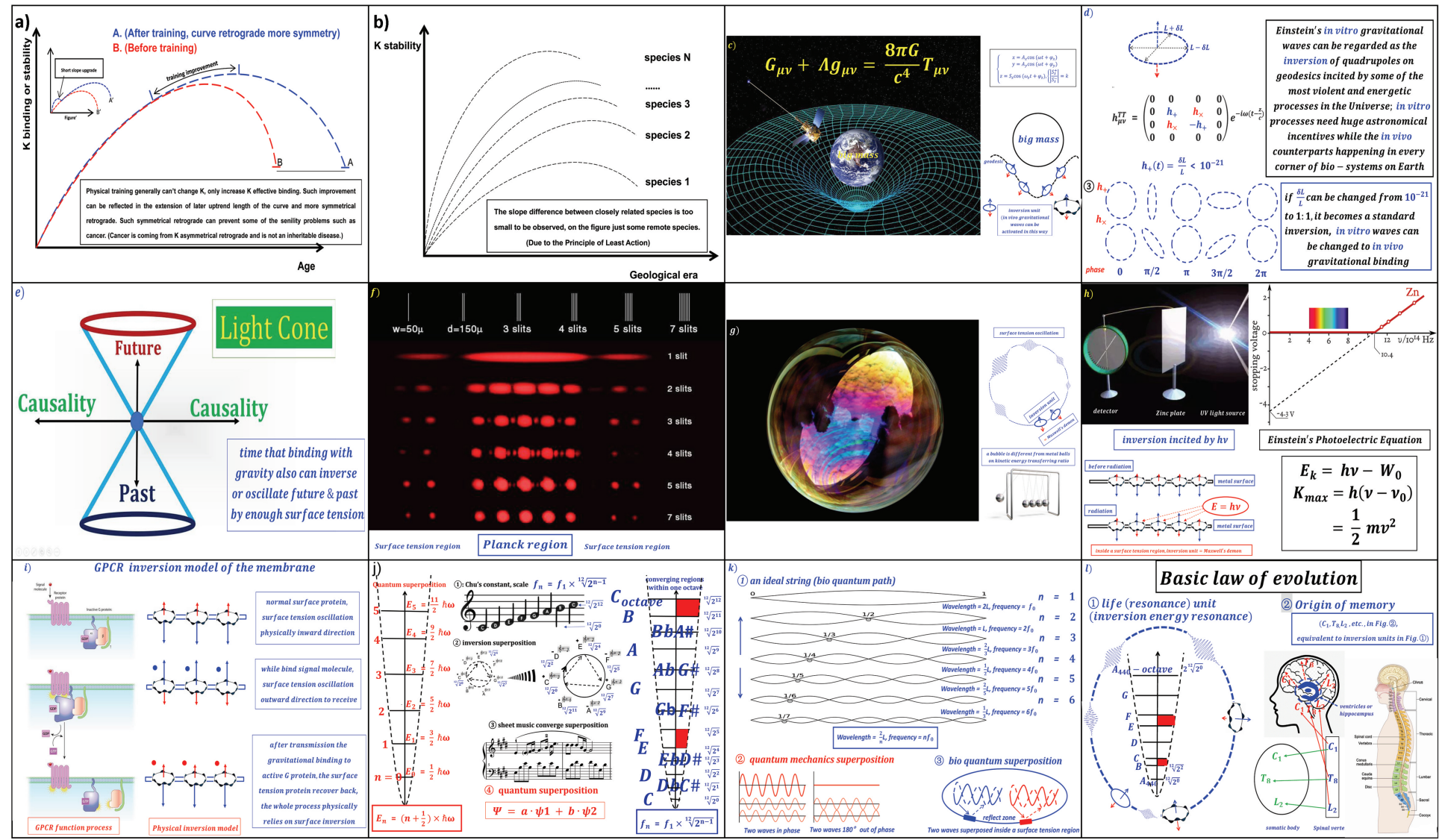

Figure 2: Inversion of Newton's Laws of Motion into the Basic Law of Evolution

a) lifetime gravitational binding curve pattern ${ }^{1}$ b) evolutionary gravitational binding pattern ${ }^{1}$ c) short of inversion in Einstein's gravitational theory d) in vitro and in vivo gravitational waves e) light cone and inversion time f) Planck regions and surface tension regions in quantum superposition g) surface tension region and its characteristics $\mathbf{h}$ ) photoelectric effect and the inversion model i) GPCR inversion model cross the membrane $\mathbf{j}$ ) the difference between the quantum mathematic superposition and music inversion superposition $\mathbf{k}$ ) bio quantum path inversion superposition under a surface tension region l) Basic Law of Evolution expressed by enclosed surface tension with part or full integrated inversion units resonate with the core bio quantum path superposition.

According to the diving coach, diving from a $10 \mathrm{~m}$ platform can be started as early as 6 years old, a lower platform such as $3 \mathrm{~m}$, can even be started at 2 years old and move to a $10 \mathrm{~m}$ platform shortly. (More generally speaking, a human baby can give birth inside water, possibly even just after birth, a baby can dive from $1-2 \mathrm{~m}$ in height after six months.) Not considering these earlier possibilities, it is still estimated $98 \%$ of humans on earth can participate in such a test unless with very serious birth defects. A free-fall state is the most optimized condition for the living being gravitational binding process, till now we still cannot find any alternative test method. Even $10 \mathrm{~m}$ water diving is dangerous for very old age people who lack preparation training, we can know the risk time point from the earlier monitor start from 6 years old for the same person and take rational precautions. At least $90 \%$ of the lifetime of people can regulate such a test if we understand

\section{Volume 10 Issue 9, September 2021}




\section{International Journal of Science and Research (IJSR) \\ ISSN: 2319-7064 \\ SJIF (2020): 7.803}

its historical role. The lifetime FHD standard curve monitor for an individual is truly critical since it governs all known and unknown biological indicators. (E.g. In immunology, there are a lot of acquired immunity indicators; however, no innate immunity indicator is available. Also, the senescence of a person is challenging to be calibrated. The only solution to understanding or quantify innate immunity is through the lifetime $F H D$ standard curve, or alternatively, by ancient Chinese Kungfu training system $(\mathrm{p} 1681, \rightarrow)^{1}$. FHD tests can also be integrated with physical training, no matter which sportive activity one person participates in, such as running, jumping, kicking a ball, etc., the biological benefit before and after a certain period of training can be indisputably calibrated by $F H D s$.) In a long period of history, the $F H D$ will be an irreplaceable indicator for medical \& sportive, theoretical \& application tests for life functions.

\section{Measuring the gravitational binding of animal sexual behavior to explore for human data}

Among all animal or human behaviors, sexual behavior should be the most impact factor on FHDs. Suppose an adult man gets $F H D s$ on the $10 \mathrm{~m}$ platform under normal conditions, after a sexual behavior, the $F H D$ data or the somatic bio-inertia will be reduced, then need a certain time to gradually recover back the gravitational binding and the recovery rate decaying with age and health condition. Such a reduction is sensitive to the $F H D$ detection even one week after the behavior. (Ancient Chinese people did not know gravitational binding and our FHD test; however, they did test the impact of sexual behavior by their Kungfu training methods for thousands of years. Our FHD test can conveniently get equivalent results to those in ancient times; offer a test that exceeds all known ancient and modern methods.) However, while suggested the human volunteers perform such a test, they all rejected it because of privacy. No one likes such a result to be exposed to other people. This reminds us, we should give personal lifetime $F H D$ standard curve enough privacy in the future application or scientific study. Also due to this reason, we have to use animals to perform the sexual impact test to replace human data as in (Tab. 8), albeit the sexual capacity and relevant parameters of human somatic bodies should be significantly higher than those of animals:

Table 8: $F H D s$ of animals before and after sexual behaviors

\begin{tabular}{|c|c|ccc|c|c|c|c|}
\hline animal & Falling height & \multicolumn{2}{|c|}{ Pre-sex $F H D(\mathrm{~cm})$} & Ave & STDEV & Post-sex FHD $(\mathrm{cm})$ & FHD sexual loss \\
\hline rooster 1 & 5.28 & 44 & 52 & 45 & 47 & 4.36 & 32 & $31.9 \%$ \\
\hline rooster 2 & 5.28 & 33 & 41 & 48 & 41 & 7.50 & 26 & $36.1 \%$ \\
\hline rooster 3 & 5.28 & 57 & 43 & 52 & 51 & 7.09 & 38 & $25.0 \%$ \\
\hline rabbit 1 & 5.44 & 38 & 33 & 28 & 33 & 5.0 & 23 & $30.3 \%$ \\
\hline rabbit 2 & 5.44 & 42 & 40 & 39 & 42 & 3.51 & 31 & $26.8 \%$ \\
\hline
\end{tabular}

*** For roosters, separately from hen to feed for 3 months, tie the wing and fall from $5.28 \mathrm{~m}$ to a children trampoline with $1.5 \mathrm{~m}$ enclosure, three repeats; after 2 hours rest to calm the animal, use chicken artificial insemination technologies to make the rooster ejaculation, then the same manner to test $F H D$ s. Rabbits use the same way, separately feeding the male animal, release 3 times, after 2 hours of calm down, supply female animal for mating, after sexual behavior, release the male animal for FHDs test. We also prepare rats and mice, however, failed to arouse their sexual behavior after release from height three times. Therefore, no data can be collected on these two animals. However, this experiment is universal for sexual behavior for all living beings including humans.

Here, we only test a few animals; however, the sexual behavior impacts on bio-inertia should be more general in animals or even the whole biological kingdom, based on thousands of years' test by ancient Chinese people with their unique Kungfu system. Compared to humans, the sexual behavior frequencies of most animals are quite less in quantity which controls more by the estrous cycle. It seems those processes such as plant proliferations or bacterial plasmid transmissions (limited sexual behavior that only refers to

\section{Results, Theoretical Section}

\subsection{The limitations of Newtonian three laws of motion for modulation bio-inertia}

In classical mechanics, Newton's laws of motion are three laws $^{3}$ that describe the relationship between the motion of an object and the forces acting on it. Newton's laws of motion, together with his law of universal gravitation ${ }^{3}$ and the mathematical techniques of calculus, provided for the first time a unified quantitative explanation for a wide range of physical phenomena, which was first published in 1687 and laid the foundation for Newtonian mechanics and modern science $^{3}$. The fundamental method that originated in this system is the isolated logic method, which means separate the around $2 \%$ of the genome) are quite different from animals in sexual behaviors, they should still be accompanied by large gravitational binding shifting, otherwise, there is no chance for evolution to happen due to the offspring cannot get a potentially higher internal motion gravitational binding than their parents. It is such a kind of gradually accumulated gravitational binding among generations in species that drives evolution.

\section{Volume 10 Issue 9, September 2021}


inertia". It states that an object either remains at rest or continues to move at a constant velocity unless it is acted upon by an external force ${ }^{3}$. It obviously supposes a world that a surface tension field never exists. For bio-inertia, we can't simply transfer that as the capability of keeping motion state as for rigid bodies. Bio-systems are to keep alive and metabolisms instead of keeping a certain motion state against a Newtonian reference frame albeit both are "keeping the inertia state". Bio-inertia is born in a non-isolated environment for bio-system survival. Newtonian inertia describes an ideal theoretical acceptable state in which a rigid object will keep its motion state in an absolute isolated environment. However, with the previous bio-inertia discussion, we still can adapt the Newtonian first law into bio-system in vivo environments like the following:

All in vivo active biomaterials or biostructures remain their state on bio quantum path(s) unless they are acted upon by its internal motions or the state inversion of relevant bio quantum paths. (Bio quantum paths represent the shortest distances or the most efficient gravitational binding functioning path of the in vivo environment. Itself is a gradient.)

Note: here the bio quantum path replaces the constant velocity on a straight line in Newton's system. With the basis of the modified first law, we then can get the modified second law:

To change the state of active biomaterials or biostructures on a bio quantum path, any force or operation must fall into this bio quantum path pattern and then can issue impacts. (The state shifting demonstrates the Le Châtelier effect for all gravitational sensitive parameters.)

This modification reminds us we can't simply impose a force on an object moving along a bio quantum path to change its state as we routinely utilize $\vec{F}=m \cdot \vec{a}$ to change the state of a non-bio quantum path running object. To change the state of an object moving on a bio quantum path, we must first make the force falls into the bio quantum path pattern, then can change its state. (The forces that are modulated by $\vec{F}=m \cdot \vec{a}$ have little impact on objects with the bio quantum path state. Only impacts from a large gravitational field plus forces that have been patterned to the bio quantum path can accumulate effects for changing states. And while impacts can accumulate, the Le Châtelier effect replaces the Newtonian acceleration of an object for the state shifting level evaluation.) Also, the concept of mass no longer fits bio-systems (the more surface tension region gravitational binding of an object, the more errors for it to use mass indicator). The concept of time is also different, must be calibrated by different surface tension gravitational binding structures. Some fashionable stories such as CRISPR/Cas9 genome editing, synthetic biology, etc., always face difficulties. The jinx is still above Newtonian second law modification; it tells us about the prerequisite requirement for changing the states of something on a bio quantum path. If we edit the linear nucleic acid sequence, only while such editing falls into the system bio quantum path superposition, then it can work; otherwise, the linear nucleic sequence editing only gets "dead DNA segments" without any biological function. The Newtonian third law states that all forces between two objects exist in equal magnitude and opposite directions. This law is still only correct for rigid bodies, just to write the modified version of this law needs to refer to some future understanding of surface tension regions, we then put that later.

Newton's law of universal gravitation ${ }^{3}$, the first inverse-square law in physics and human history, got much insight from Kepler's laws of planetary motion. Therefore, it can contribute greatly to astronomy and the discovery of planets. (Actually, we can note that Kepler's law has included relative mobility components; just Newton's later equation shields these components.) However, transfer from astronomy pattern to universal beings on earth with an inverse-square formula, later established Cavendish or other experiments only comply with original Newtonian $\mathrm{G}$ format, never prove that such a transfer is yet "universal". From our previous experiments, at least for bio-systems, the inverse-square gravitational law will fall in quite an intolerable discrepancy. If people still like to transfer from astronomical conditions to existing on earth, we have suggested modifying the original equation to $\vec{F}=K^{N} \cdot G \frac{m \cdot M}{r^{2}},\left(K^{N}\right.$ is used to calibrate the $10^{9}$ shifting) for some in vivo conditions $(\mathrm{p} 1684, \rightarrow)^{1}$.

Albert Einstein had made substantial modifications to Newtonian mechanics; however, still rarely referred to the shifting of the $\mathrm{G}$ range on a large scale. He only adopted the original format of inverse-square law into Poisson Equation: $\nabla^{2} \Phi=4 \pi G \rho$ (here, $(r)=-G \frac{M}{r}$ ), and then further infer his field equation. Under nonrelativistic conditions, the results from his theory are still the same as that of Newton's. Therefore, for bio-inertia that can't be "universally" included in the Newtonian equation, Einstein's theory equally can't handle with.

\subsection{The limitations of Einstein's equivalent principle for modulation bio-inertia}

We have referred to Einstein's elevator thought experiment used to justify inertia mass = gravitational mass before. We can understand the prerequisite condition of this equivalence is that there is no exchange between inertia mass and gravitational mass. For rigid bodies or space-time that not moving along a bio quantum path, this prerequisite condition roughly exists. For non-rigid bodies or a space-time patterned by bio quantum paths (or superposition) which is called bio curved space, physical laws can no longer be equivalent. As mentioned before, we can't use the conservation of momentum between a non-living object and a living organism. The quiddity of this problem still traces back from the isolated logic method established in Galileo and Newton's time. We can "isolated logically" separate the research target from an environment and no need to consider the corrections between this target and the environment. However, for bio-systems which inseparable from the environment, the isolated logic way will unavoidably deprive of surface tension functions.

The left of (Fig.2c) shows Einstein geodesics that modulate gravity is entirely geometrical by nature (that is, the metric alone determines the effect of gravity) and does not have any extra fields associated with it. This geometrical gravity is somewhat different from internal motion gravitational binding, not only in scale (astronomical VS individual organism), gravitational strength $\left(10^{9} \times \mathrm{G}\right)$, relativistic 
condition, and time, but also critically in inversion (p1694, $\rightarrow$ )$^{1}$ processes. At the right of (Fig.2c), we added some inversion units on the geodesics that previously modulate massive object curves space and a light object moving along the geodesic. Einstein never described that gravity could inverse perpendicularly along the geodesics as shown in the bottom sketch. If we reluctantly regard Einstein's quadrupole polarization which issues in vitro gravitational waves as a kind of special inversion that can happen along the geodesics, the level of which is still too weak. (Einstein himself never believes gravitational waves can produce in such a way. As in (Fig.2d), for the quadrupole that issues Einstein's gravitational waves, $\delta \mathrm{L} / \mathrm{L}=10^{-21}$, for that to change to an inversion process does need " $\delta \mathrm{L} / \mathrm{L}$ " to be enlarged to a level close to a range lower than $1: 10$. This means that Einstein never realized any inversion process or similar modulation that is inseparable from surface tension forces.)

Einstein's gravitational waves are disturbances in the curvature of space-time, generated by accelerated masses that propagate as waves outward from their source at the speed of light. Newton's law of universal gravitation does not provide for their existence, since that law is predicated on the assumption that physical gravity propagates instantaneously (at infinite speed), albeit Newton himself didn't satisfy with such kind of instantaneousness. Inside bio-systems, there also exist in vivo gravitational waves which are totally different from Einstein's gravitational waves. The speed is lower (estimated not far away from nerve conduction velocity of $120 \mathrm{~m} / \mathrm{s}$ ), and the function range only in vivo or slightly goes outside the organism (estimated less than 100m). These types of gravitational waves which originate from bio quantum path superposition or inversion, shape the in vivo functioning, and are impacted greatly by almost all in vivo active ingredients, are quite different from the in vitro Einstein gravitational waves that defined as "ripples" in space-time caused by some of the most violent and energetic processes in the Universe, which rarely impacted by the things it propagates by. (Einstein never realized that his gravitational waves impact or be impacted by a surface tension region greatly. Such an impact needs aether under the surface tension region. The results of Einstein's gravitational waves have given up the concept of "aether" albeit he theoretically believes in it. In bio-systems, the in vivo gravitational waves are "aether", human CSF is such a kind of in vivo aether with the highest gravitational binding.)

(Fig. 2e) shows Einstein Light cone, the time in the past and future are separated. However, in bio-systems, sometimes these two parts can partially inversion. Organism lifespan is a kind of inversion time that originates from surface tension region(s).

2.3. The limitations of Schrödinger wave function for modulation bio-inertia, and the unavoidability of inversion protection for the wave function in a non-isolated environment

Quantum mechanics, albeit originated from macrocosmic measurable Planck's law of black-body radiation, only modulates the physical properties of nature at the scale of atoms and subatomic particles. There are no convincing explanations why a discipline based on energy can only be restricted into the microcosmic world, and the only jinx for the macrocosmic world seems on the experiment.

The Schrödinger equation is a linear differential equation that governs the wave function of a quantum-mechanical system, which assumes that if two wave functions $\psi 1$ and $\psi 2$ are solutions, then so is any linear combination of the two: $\Psi=$ $\mathrm{a} \cdot \psi 1+\mathrm{b} \cdot \psi 2$, where $\mathrm{a}$ and $\mathrm{b}$ are any complex numbers normalized by $|a|^{2}+|b|^{2}=1$. This linearity axiom has experienced Planck's black body, Einstein's photoelectric effect, till Schrödinger wave function. It has been regarded as the most charming characteristic of quantum mechanics since a quantum system can have two classical states, one particle can have different positions, different energies, etc. However, if we carefully watch (Fig.2f), a series of multi-slit interference experiments, we can easily find the linear region is not unlimited or can be existed independently; always linear region enclosed or interweaved with the non-linear region. This should be a very general experiment, no matter we use light, water, mechanical wave, or any method, once interferograms are present, we always can find the above two types of inseparable regions. Now we can simply call the linear region as "Planck region", which can be written as $E=$ $h v$ and have also been studied extensively by conventional quantum mechanics; the non-linear region as "surface tension region", which has been neglected by quantum mechanics, or more precisely, covered by quantum mechanics probability and then no attention (all surface tension regions are intrinsically unsymmetrical, can't be existed flat; unsymmetrical properties originate inversions and structure resonance.). These two regions are inseparable in a non-isolated environment albeit conventional quantum mechanics is established under the assumption that the Planck region can exist independently. Nowadays quantum mechanics only can deal with non-living beings, the fundamental reason is that it restricts itself to the Planck region with probability and neglects the surface tension regions; however, life functioning must rely on the interaction between the Planck region \& surface tension regions.

Wave function collapse is another much-debated problem of this discipline. Wave function collapse occurs when a wave function-initially in a superposition of several eigenstatesreduces to a single eigenstate due to interaction with the external world. This interaction is called an "observation". Substantial reports are challenging this story. E.g. Calculations of quantum decoherence show that when a quantum system interacts with the environment, the combined wave function of the system \& environment continues to obey the Schrödinger equation ${ }^{8}$, as decoherence does not reduce to a single eigenstate ${ }^{9}$. However, to fully understand this problem we possibly can go back to the surface tension regions that cover Planck's regions. The left of (Fig. 2g) shows a soap bubble maintains by Minimal surface tension, a colorful film. The right top of (Fig. 2g) draws the oscillation and inversion of the film; compare to the right bottom of (Fig.2g) we could understand the energy characteristics between two types of objects. The solid metal ball can easily transfer holistic kinetic energy to each other by the law of the conservation of momentum. The holistic kinetic energy item $\frac{1}{2} m v^{2}$ even can be almost $100 \%$ transfer from one end to another end for the metal ball queue with 5 members in the figure since these balls have no surface tension regions to shift

\section{Volume 10 Issue 9, September 2021}


the momentum process. For the soap bubble, the transferring efficiency of kinetic energy is quite lower. However, it is difficult to get what in the bottom figure of the transferring efficiency for kinetic energy even for two bubbles. The fundamental cause is still the inversion; a surface tension region can transfer the kinetic energy into potential energy by inversion. There are inversion units in the right top figure, these inversion units compose of one inward vector (blue) and one outward vector (red) with a net inward vector to maintain the surface tension. Inversion means the blue vector and red vector exchange each other in response to environmental impacts; this is a type of frequency energy. Soap bubbles can't transfer kinetic energy in a higher efficiency to each other, that is due to the surface tension inversion can transfer the kinetic energy into potential type energy in higher efficiency. In contrast, the efficiency of metal balls to transfer kinetic energy to potential energy is almost zero. We then know the general function of all surface tensions: transfer kinetic energy to potential energy. Also due to the oscillation, the bubble film can bind gravity. The bubble only has one layer of surface tension region; therefore, gravitational binding only shifts and inversion around the film layer. Unlike those seen in a rainbow, which arises from differential refraction, the colors are seen in a soap bubble arise from interference of light reflecting off the front and back surfaces of the thin soap film, which is also an inversion process. We can even say that gravitational binding inversion lets us see a colorful soap bubble film. In conventional quantum mechanics, the wave function is not a bound state in an isolated environment. The energy levels come from the energy intrinsic properties instead of the wave function itself. However, for a wave function in a non-isolated environment, it is indeed in a bound state with inversion protection.

The photoelectric effect is also mechanized by surface tension inversion $(\mathrm{p} 1692, \rightarrow)^{1}$. As in (Fig.2h), the process is the emission of electrons when electromagnetic radiation hits a material, get results that disagree with classical electromagnetism. In 1905, Einstein proposed that light consists of tiny packets of energy $h v$ known as photons. The maximum kinetic energy of the electrons is:

$$
K_{\text {max }}=h\left(v-v_{0}\right)
$$

Einstein's formula, however simple, not only explained all the phenomenology of the photoelectric effect but also had far-reaching consequences in the development of quantum mechanics. This process has already included inversion as in the left bottom of (Fig.2h) albeit people rarely realize that. The electrons on the metal surface compose of oscillation energy with inward vector (blue) and outward vector (red), once the photon $h v$ hits the inversion unit, the inward and outward vector change each other and release current. This way follows the principle of least action to deliver electrons to currents. Such an inversion model agrees with photoelectric results since the response time is lower than $10^{-9}$ seconds. It is a very general characteristic of all surface tension regions; interaction with the external world by means of inversion, here the inversion processes are the manner to comply with the principle of least action. (From here we could also find the weakness of thermodynamics, this theory suggests an isolated system for its second law. However, for any ideal isolated system, once boundary presents, it has inversion units; no matter inversion unit(s) is more or less, there exists! The second law of thermodynamics needs a surface tension region with zero inversion, or all the inversion units with equal blue and red vectors, which is impossible to present in the real world. We even can regard inversion units as a group of Maxwell's demons, which mechanisms we'll refer to later.)

Quantum entanglement was first presented in Einstein's paper as EPR paradox ${ }^{10}$. An entangled system is defined to be one whose quantum state cannot be factored as a product of states of its local constituents; that is to say, they are not individual particles but are an inseparable whole. In entanglement, one constituent cannot be fully described without considering the other(s). It is entangled if this sum cannot be written as a single product term. This means the entangled state is a very general inversion mix with random and musical types of inversion. Experiments have validated entanglement at a large distance to the satellite orbit; however, still not correlates the process with gravity. We believe that not only quantum entanglement must rely on gravity but also wave function can't be exempt from gravitational binding. With gravity as a mechanism, quantum entanglement is no longer adapts to Einstein's comment "spooky action at a distance" since it is so general.

In bio-systems, $\mathrm{G}$ protein-coupled receptors (GPCRs) form a large group of evolutionarily related proteins that are cell surface receptors that detect molecules outside the cell and activate cellular responses. $G$ protein-coupled receptors are found only in eukaryotes, including yeast, choanoflagellates ${ }^{12}$, and animals. The ligands that bind and activate these receptors include light-sensitive compounds, odors, pheromones, hormones, and neurotransmitters, and vary in size from small molecules to peptides to large proteins. GPCR demonstrates a perfect inversion model as in (Fig.2i), which possibly includes many subordinate inversions, evolution from simple molecules to large seven cross-membrane complexes still comply with the cross-membrane inversion pattern.

Now, back to the wave function collapse; in quantum mechanics, the wave function is claimed as complex-valued probability amplitude, and the probabilities for the possible results of measurements made on the system can be derived from it. The wave function is a function of the degrees of freedom corresponding to some maximal set of commuting observables. $|\psi|^{2}$, is a real number interpreted as the probability density of measuring a particle as being at a given place - or having a given momentum - at a given time and possibly having definite values for discrete degrees of freedom. Most fundamental experiments such as the single slit, double slits, etc. are happening in a natural environment that is never isolated. The application of Schrödinger's equation to an open system in the present sense concerns the relative robustness of wave function, no matter the range of structure. Wave function collapse concerns such robustness in an environment. No matter at any conditions gives a surface tension with inversion to the outside range of the wave function will increase its robustness or extend the wave function collapse to a countable process. The conventional wave function collapse model is only a compromised choice that while lacking the inversion region outside the wave function, not happens in the real world. Our previous mutation experiments $1.4,1.5$ still could be modulated as the mutation of the wave function. For the half bottle oil or water, in the

\section{Volume 10 Issue 9, September 2021}


process of falling, the liquid oscillates with the bottle wall. This is a kind of oscillation energy that can be regarded as a wave function with the bottle wall as the inversion protection barrier. Wave function satisfies the equation: $\widehat{H}=\widehat{T}+\widehat{V}$, due to the wave function of the oil bottle is different from that of the water bottle, the gravitational binding is different, we then can see the $F H D$ between them. For living being mutations, such as mutations 3, 4, 5; still equivalently modulated, just add some conditions: $\widehat{H}=\widehat{T}+\widehat{V}, \widehat{V} / \widehat{T}=\mathrm{k}$, (as mentioned, living being can high-efficiently transfer the kinetic energy into potential energy, then there is a $\mathrm{k}$ restriction in addition to the Hamiltonian. Living beings transfer efficiency comes from multi-surface tension regions). The $F D H$ s between the living being VS non-living being, or a person's healthy VS Sub-health state, etc., all due to the difference in wave function gravitational binding in the free fall process. There is no gravity parameter in conventional Schrödinger's equation:

$$
\begin{aligned}
& \widehat{H}=\widehat{T}+\widehat{V}=\frac{\hat{p} \cdot \hat{p}}{2 \mu}+V(\boldsymbol{r}, t)=-\frac{h^{2}}{2 \mu} \nabla^{2}+V(\boldsymbol{r}, t) \\
& E \psi(\boldsymbol{r}, t)=-\frac{\hbar^{2}}{2 \mu} \nabla^{2} \psi(\boldsymbol{r}, t)+V(\boldsymbol{r}) \psi(\boldsymbol{r}, t)=i \hbar \frac{\partial}{\partial t} \psi(\boldsymbol{r}, t)
\end{aligned}
$$

The higher levels of gravitational binding in our free-fall mutations should come from the complex surface tension inversion regions compose of the bio-systems. There is another equation in quantum mechanics known as Dirac equation $^{11}$ :

$$
\left(\beta m \mathrm{c}^{2}+c \sum_{\mathrm{n}=1}^{3} a_{n} p_{n}\right) \psi(x, t)=i \hbar \frac{\partial}{\partial t} \psi(x, t)
$$

Dirac's purpose in writing this equation was to explain the relativistic moving electron, and so to allow the atom to be treated in a manner consistent with relativity. It predicted negative-energy quantum states for relativistic electrons and later be explained as the Dirac Sea. We believe the quiddity of negative-energy states is also a kind of special inversion which equivalent to the photoelectric effect or other inversions. That means even in a discipline that lacks an inversion model, we still can find some inversion trace evidence. (We should note, both dark matter and Massenergy equivalence are easily misleading. Even so-called dark matter can present, and while it meets with other non-dark matter, the energy can be released still quite limited since the time for the energy process is non-unitary; therefore, restrict the theoretical transfer efficiency between matter and energy. For this reason, the Dirac equation only means energy inversion, never means the evidence for dark matter.)

Applied inversion superposition in quantum mechanics also needs to break the normalized conditions. It is a restriction on the allowed evolution of quantum systems to ensure the sum of probabilities of all possible outcomes of any event equals 1 , $\int_{-\infty}^{+\infty} \Psi^{*}(x) \Psi(x) d x=\int_{-\infty}^{+\infty}|\Psi(x)|^{2} d x=1$. For bio-systems, a biomaterial's normal state and its inversion state can no longer be normalized into 1 , time is inversion time and not the unitary time in conventional quantum mechanics. It is due to time in bio-systems is inversion time which correlates with the environment deeply; therefore, time travel is impossible, it will damage the bio-system first before travel is fulfilled. There are still other problems for quantum mechanics to adapt to bio-systems which we will refer to later. We can simply call the wave function in conventional quantum mechanics in vitro and for bio-systems as in vivo wave function. The critical difference between in vitro and in vivo wave function is mathematic superposition in conventional quantum mechanics and the inversion superposition in bio-systems.

\subsection{From non-living being mathematic superposition in conventional quantum mechanics to bio-system surface tension inversion superposition that possess universal recovery capability}

Under in vitro conditions, without considering application range, also neglect the parameter- against-gravity-recovery accumulation process detail, Schrödinger wave function and Einstein's gravitational waves are the same sorts of oscillation energy if we accept wave function can bind gravity. Our testing of the FHDs for living beings by free-fall could be regarded as test the in vivo Schrödinger wave function or in vivo Einstein's gravitational waves, the quiddity of which is still internal motion or inversion of parameters against gravity. Under a free-fall state, gravitational binding can be calibrated efficiently. We have suggested using Chu's constant in quantum mechanics, that is to take $\left|E_{n}\right\rangle=$ $\sqrt[12]{2^{\mathrm{n}-1}} h v$ to replace $\left|E_{n}\right\rangle=n h v(\mathrm{p} 1692, \rightarrow)^{1}$, also suggested frequency notation $(\mathrm{p} 1709, \rightarrow)^{1}$. (Music scale can be written as $\left|f_{n}\right\rangle=f_{0} \times \sqrt[12]{2^{\mathrm{n}-1}}$, omit $f_{0}$ of $440 \mathrm{~Hz}$ tuning frequency and then can get that notation.) Now, we should understand the fundamental difference between quantum superposition and music scale frequency inversion converge superposition (which is the basis of life memory we'll explain later):

1) Quantum mechanics is mathematically modulated in an isolated system and music happens in a non-isolated system. As a consequence, quantum never clarifies its resonance characteristics, the musical system must rely on resonance. And quantum state collapse is even a fully anti-resonance modulation.

In conventional physical theory, resonance describes the phenomenon of increased amplitude that occurs when the frequency of a periodically applied force (or a Fourier component of it) is equal or close to a natural frequency of the system on which it acts. When an oscillating force is applied at a resonant frequency of a dynamical system, the system will oscillate at higher amplitude than when the same force is applied at other, non-resonant frequencies. However, such a model originated from a rigid system only correct while oscillation transfer only restricted to certain research targets or no extension of the oscillation. In a non-isolated environment, the resonance can be spreading widely which certain inversion becomes inseparable. A music compositing process can be regarded as the macrocosmic superposition of scale note frequency: $\left|f_{n}\right\rangle=f_{0} \times \sqrt[12]{2^{\mathrm{n}-1}}$, and accumulate the inversion resonant driving forces that come from each superimposed frequency. In music, if we hope to superpose scale note(s) into a piece of music, that will never be such a linear sum up, the processes of inversion are totally indispensable. The quality of a musical instrument or its combinations relies on the inversion resonant capability between each scale level: $\left|f_{n}\right\rangle=f_{0} \times \sqrt[12]{2^{\mathrm{n}-1}}$, tension among scale levels fully comes from the resonant cavity of the instrument. However, quantum mechanics never believe the robustness of interference fringes comes from resonance.

2) A large part of quantum mechanics describes the movement of electrons, there are limitations for particle scale it can deal

\section{Volume 10 Issue 9, September 2021}


with, compare to music can deal with almost all types of oscillations driving tendency, quantum mechanics only can deal with a few types of particles with probability and a limited number of parameters. Without considering such a description range, what critical is the linear quantum superposition is totally different from the inversion converge superposition in music, and such inversion superposition is what is living organisms fully rely on.

As mentioned, quantum superposition is a kind of linear process: $\Psi=\mathrm{a} \cdot \psi 1+\mathrm{b} \cdot \psi 2$. This linearity is prided of by the Schrödinger equation since it can change complex processes into linear processes. However, such a linear process is a mistake in the music scale superposition; the latter needs the inversion superposition instead of the "allele superposition". Suppose $\left|\phi_{n}\right\rangle$ and $\left|\psi_{n}\right\rangle$ need to be superimposition, quantum mechanics always takes $\phi_{1}$ with $\psi_{1}, \phi_{2}$ with $\psi_{2}, \ldots$, and $\phi_{k}$ with $\psi_{k}$, for superposition. Music takes $\phi_{1}$ with $\psi_{n}, \ldots$, and $\phi_{n}$ with $\psi_{1}$, this is a full inversion; or $\phi_{k}$ with $\psi_{j}$, however, compensate with some inversion combination of $\psi_{j}$, this is a partial inversion. (Existing of partial or full inversion means system time is non-unitary.) No matter partial inversion or full inversion, no inversion means the stop of music; or in life, stop inversion means the end of the life function. The left hand of (Fig.2j) and (Fig.2j(4) are quantum superposition (with red color). (Fig.2j(1)) shows the scale and Chu's constant $(\mathrm{p} 1692, \rightarrow)^{1}, \quad\left|f_{n}\right\rangle=f_{0} \times \sqrt[12]{2^{\mathrm{n}-1}} . \quad\left(f_{0}\right.$ is the tuning frequency, which is widely used with $440 \mathrm{~Hz}$, omits it we then get the frequency notation $\left.\left|f_{n}\right\rangle=\sqrt[12]{2^{\mathrm{n}-1}}\right)$. As previously our modification of Newtonian first law, we could regard a scale in the right hand of (Fig.2j) as the static state.

With the scale as a relatively static state, any musical note combination different from the scale sequence in (Fig.2j(1) can be defined as dynamic. The "dynamic" of a note means it possesses a tension against the scale or certain note combinations. Such tension is usually incorporated in a chord or a movement. (Fig.2j(2)) shows the simplest cord: a major triad composed of $\mathrm{C}\left(\sqrt[12]{2^{0}}\right)-\mathrm{E}\left(\sqrt[12]{2^{4}}\right)-\mathrm{G}\left(\sqrt[12]{2^{7}}\right)$ that comes from a scale. All the three notes, C, E, G can fall on the root position of the triad structure, different root position represents different inversion. E.g. C-E-G is the primary triad, E-G-C, $1^{\text {st }}$ inversion, G-C-E, $2^{\text {nd }}$ inversion. The tension of a note against a "static" scale is dynamic in such a way. If we regard composing of sheet music as a kind of superposition, then the superposition of the same pitch or frequency still results in the same frequency, however, need to compensate with the above tension. We superimpose an $E\left(\sqrt[12]{2^{4}}\right)$ in one movement with another $\mathrm{E}\left(\sqrt[12]{2^{4}}\right)$ from another movement (these two notes must with different vector directions such as $|\uparrow\rangle,|\downarrow\rangle$ to superposition), the resulted frequency still $\sqrt[12]{2^{4}}$ which frequency keeps the same; however, must compensate with the inversion tension structure of another $\mathrm{E}\left(\sqrt[12]{2^{4}}\right)$, such an inversion structure could be a chord or chord combinations, or movements, etc. (whether this superposition changes the $|\uparrow\rangle,|\downarrow\rangle$ of $E\left(\sqrt[12]{2^{4}}\right)$ depends on the superposed inversion structure.) (Fig.2j(3) shows a piece of sheet music, due to a pair of same pitches or frequencies after superposition can omit one, then it composes of a melody and an accompaniment. All sheet music, no matter which style, is composed down from inversion superposition in this way which is totally different from quantum superposition. Music offers us great tools for modulating inversion. In the modern scientific kingdom, there is almost no tool for modulating inversion. Among all scientific isolated logic disciplines, quantum mechanics is closest to inversion processes than all others; however, it still chooses to fix inversion. The commutation relationship in quantum mechanics can be regarded as a type of compressed or "isolated logic" fixed inversion relationship.

Given a pair of operators follow $[\widehat{F}, \hat{G}]=\hat{F} \hat{G}-\hat{G} \hat{F}$, if $[\widehat{F}, \widehat{G}]=0$, they are commutation; if $[\widehat{F}, \widehat{G}] \neq 0$, they are not commutation. A typical example in quantum mechanics is, $\left[\widehat{\boldsymbol{x}}, \widehat{\boldsymbol{p}_{\boldsymbol{x}}}\right]=\boldsymbol{i} \hbar$, means they can't be measured simultaneously, that is the uncertainty principle. It also means the commutators have a common eigenvalue which can apply to quantum mechanics. The non-commutative item " $i \hbar$ " is then what quantum mechanics can target. (In classical physics, all observables are communitive, or commutator equals zero. Then the item " $i \hbar "=1$, or $\{x, p\}=1$ )

The equations of commutators in classical quantum mechanics can be given as:

$\left[\hat{A}, \widehat{A^{n}}\right]=0, \mathrm{n}=0,1,2 \cdots \cdots \quad \mathrm{K}, \quad[\hat{A}, \hat{B}]=-[\hat{B}, \hat{A}]$

$[\hat{A}, \hat{B}+\hat{C}]=[\hat{A}, \hat{B}]+[\hat{A}, \hat{C}], \quad[\hat{A}+\hat{B}, \hat{C}]=[\hat{A}, \hat{C}]+$ $[\hat{B}, \hat{C}]$

$[\hat{A}, \hat{B} \hat{C}]=\hat{B}[\hat{A}, \hat{C}]+[\hat{A}, \hat{B}] \hat{C}, \quad[\hat{A} \widehat{B}, \hat{C}]=\hat{A}[\hat{B}, \hat{C}]+$ $[\hat{A}, \hat{C}] \widehat{B}$

$[\hat{A},[\hat{B}, \hat{C}]]+[\hat{B}[\hat{C}, \hat{A}]]+[\hat{C}[\hat{A}, \hat{B}]]=0$

Fixed commutation not only induces the uncertainty principle but also quantum collapse becomes unavoidable since this discipline lacks series of the intermediate states between the quantum state and a full collapsed state, all the intermediate states must collapse instantly without any inversion. Two commutators can't be measured simultaneously in quantum mechanics. However, in bio-systems, an inversion induces the stability or recovery capability of the next levels of inversion. Different structures always need to "connect with" each other by inversions to maintain recovery capability. This is indeed a "measurement", one inversion measures a lower level of inversion, no matter which degrees of inversions. Therefore, the inversion of bio-systems or even non-living surface tension regions will be similar to the commutation relationship in classical quantum mechanics. The way in which quantum mechanics takes $\left[\widehat{\boldsymbol{x}}, \widehat{\boldsymbol{p}_{\boldsymbol{x}}}\right]=\boldsymbol{i} \hbar$ to express commutator(s) is like music takes a combination of notes (such as chords or movements) to express the inversion superposition between notes, just quantum mechanics "compress" the inversion process into commutator(s) and lacks methods to unfold them into inversion.

As in the left of (Fig.2j) for quantum superposition, if one electrons transmission between two levels, the energy shifting is $\hbar \omega$ and this $\hbar \omega$ as a step only concerns this electron. Go to the right hand of (Fig.2j) for music inversion superposition; if a note shifts one level, we can't simply say the shifting is one step $\sqrt[12]{2^{n-1}}$ since this step possibly connects with a complex combination of notes to compensate for its inversion. A note shifts for one step must drive all the relevant notes supply inversion tension to this note also follow the shifting. (We can understand this from the human CSF tap process; people who 
perform this test must keep body posture for at least two hours without moving. Because we tap the CSF from one spine, it never means the human body only loss this potion of CSF liquids. All body structures that inversion superposed with these CSF will get impacts or loss gravitational binding then must get enough rest. This somewhat likes what happens when we remove a note from sheet music. The impact of the removal is structural, not just a certain note position.) In music, the probability is almost no use, given a few notes, even if we can "calculate out" the probability of next notes or note combinations, we still can't compose sheet music with these probabilities. Sheet music needs a holistic structure, can't be expressed as probabilities between notes. For quantum superposition, the superposition round number is unlimited since there is no recovery requirement for its non-inversion superposition.

Albeit through the work of Max Planck, Albert Einstein, Louis de Broglie, Arthur Compton, Niels Bohr, and many others, the so-called wave-particle duality in quantum mechanics is still just a compromise which implies all particles study by the discipline exhibit a wave nature and vice versa $^{13}$. However, it seems music harmonics has solved this problem well than the discipline centuries ago. As in (Fig.2j3) for sheet music superposition, the up line is a melody, and the lower line is the accompaniment. For each melody note, accompaniment composes of the combinations of notes use to release the inversion tension against the static scale. We can't simply say the note shows particle characteristics and the combination which uses to release its inversion tension possesses wave characteristics. The movement possesses wave-particle duality. It is groundless to say a musical note is a wave, a particle, or a duality, only while it is integrated into a piece of sheet music, we can know it is issuing which role base on the tension release function of that section. This type of wave-particle duality seems different from what quantum mechanics storied. A lot of quantum mechanics textbooks use De Broglie matter-wave $\lambda=h / \mathrm{mv}$ to claim that the wavelengths of macrocosmic objects are so short, then unobservable. This is an unconvincing story. If we take the music system, a human does show wave-particle duality. CSF is the wave function of the holistic somatic body, and locally on each part of the body, we still can find diverse subordinate wave-particle dualities. By means of multi-surface tension regions, the wave-particle dualities of life have been enlarged to a scale that far beyond what a matter-wave formula can include, and the ancient China CSF-ligament Kungfu training system does sustain most of the bio-systems facts. We can say that music "unintentionally" supplies modulation tools better than all extant physical theories including conventional quantum mechanics for life modulation.

\subsection{Inversion of Newtonian third law into the Basic Law of Evolution}

In quantum mechanics, the round (copy) number that a series of energy levels can be subsequently superposed is axiom into unrestricted. This is possibly why quantum mechanics claims that the discipline only deals with an isolated environment. As mentioned, the surface tension region and Planck region are inseparable for superposition in a non-isolated environment. Then for each round of superposition in Planck's region, it should accumulate impact from the surface tension region of that round of superposition, no matter such an impact is high or lower. Finally, for superposition in a non-isolated environment, the round (copy) number which a quantum state can be subsequently superposed will rely on gravitational binding and cannot be unrestricted. (Later we can understand, the five postulates of conventional quantum mechanics are present for probability justification, they contradict with the bio-system inversion superposition from the very beginning.) We have written down the modifications of Newtonian $1^{\text {st }}, 2^{\text {nd }}$ laws before, now with previous discussions we can modify the $3^{\text {rd }}$ law into the Basic Law of Evolution as follow:

Bio quantum path duality equivalents the quantizing time or memory between Planck regions and surface tension regions by a string topological property equation: Wavelength $=\frac{2}{n} L$, frequency $=$ $n f_{0}$, to drive or proliferate the duality system.

The literal meaning of the statement: 1) the Planck regions and relevant surface tension regions are inseparable for bio quantum path superposition. 2) memory is a kind of negentropy that is essential for life functioning and evolution. From the lower evolved species till advanced one, even for biomaterials, such as a DNA segment, etc., all need memory that originated from surface tension for active functioning. Memory was born at the start of life, never like people's belief that memory only happens in advanced species. (The term "negentropy" comes from Schrödinger who initiates but lacks modulation of it. Later, we can see that "memory", "negentropy", "inversion energy", "surface tension energy" and even "quantizing time" etc., follow the same modulation called "life unit". Here, the term "memory" is a general term that includes the fundamental origin, not just the human memory format we usually see. From here, we can even say that inversion energy is the kind that possesses memory function, human memory we usually know is only the advanced format that bases on such origin.) 3) Under the same surface tension or in vivo environment, the structures acquired with more inversion round numbers will possess more negentropy or gravitational binding than those similar structures with less inversion round numbers. All primary biological processes or structures are evolutionarily made to be bio-reversible to "resist" the shifting of the environment, which tend to Chu's constant scale: $\left|\sqrt[12]{2^{n-1}}\right\rangle$ with the increasing of surface tension regions. 4) the Newtonian 1st Law describes an ideal theoretical acceptable state in which a rigid object will keep its motion state without outside impact in an absolute isolated environment, such a state does not exist in the real world. Bio-systems can get a compromised condition, not without outside impact, just outside impact has minimum impacts on the system or such an impact only happens on the peripheral structures and can't reach the inner Chu's constant structures. We call it the "minimum outside impact state", such a minimum outside impact state equivalent to the inner core bio quantum path maximum inversion state. Or we can say, while no outside impact, Newtonian objects keep their previous motion states, and bio-systems equivalently keep the maximum bio quantum path inversion state. This is what the modification of the 3rd law means. (From here we also can understand the fundamental difference between rigid objects and enough surface tension bio-systems.) Now we can go to further detail:

\section{Volume 10 Issue 9, September 2021}


As mentioned in the previous modification of Newtonian $2^{\text {nd }}$ law, anything that can change the state of a bio quantum path must follow its pattern; those who fail the pattern cannot impact the things moving on a bio quantum path. The reason is due to the surface tension region(s) of bio quantum path(s) albeit we omitted that detail at that time. Now the modification of the third law comes just back to the omitted surface tension functions. There are two prerequisite key points:

First, the bio quantum path(s) of a system is the oscillation energy that the whole system tends to the recovery capability of an ideal oscillation string. As in (Fig. 2k(1)), for an ideal string, the oscillation length and frequency relation should follow the same pattern no matter we move upstream or downstream unlimitedly (upstream means in the figure, we connect 2 strings get $4 \mathrm{~L}$ wavelength or connect $\mathrm{n}$ strings get $2 \mathrm{~nL}$ wavelength, etc.; downstream means hedges the string in $\mathrm{n}$ sections.), this is an ideal string. In the real condition, due to the impacts of the environment or the restriction of inversion protection of an ideal string, a string only can effectively follow the formula: Wavelength $=\frac{2}{n} L$, frequency $=n f_{0}$ in a certain range. E.g. upstream 5 lines and downstream 6 lines fall into acceptable recovery rate. We can define this range as the actual recovery range of a string. This recovery range modulation adapts for both a musical string as well as all in vivo active biomaterials, such as DNA, RNA, protein, etc. (A string issues mechanic waves; however, in vivo gravitational waves still follow the same inversion pattern that is based on surface tension regions; therefore, we can use the same music patterns to modulate in vivo gravitational waves, with no need to differ them.)

Second, the evolution of inversion superposition is to acquire the recovery capability of a bio-system by inversion. (Fig. $2 \mathbf{k}(2))$ shows the wave or quantum mechanics superposition in conventional physics, two wave sources in phase get constructive interference, and two wave sources are $180^{\circ}$ out of the phase, get destructive interference. The conventional quantum mechanics superposition only refers to the Planck region; disciplinarily "neglect" the inseparable surface tension region as shown in (Fig.2f). Via evolution, all bio-system superposition become inversion superposition. As in (Fig. 2k(3)), two waves (red \& blue solid lines) need to superpose inside an enclosed surface tension region. They will first interact (reflect) with the surface tension region (reflective zones) to get their inversions (red \& blue dotted lines), then superposition by the inversed waves (blue $\&$ red dotted lines). This type of inversion superposition is in a lower probability and weak strength to happen for non-living beings (due to very lower inversion energy available); however, becomes the fundamental superposition manner inside living beings. Life evolution takes geological time to involve the inversion superposition which must base on surface tension is to acquire the recovery capability that is essential to life. In physics, waves are generally expressed into a Cosine/Sine wave, these curves are symmetric. While we constructed the rigid bio quantum path in (Fig.1j) to show parameter-againstgravity-recovery accumulation, we still use symmetric format, but it has already included polarized or asymmetric gravitational binding. We modify the term of wave(s) into bio quantum path(s), since after an effective surface tension region is presented and functioning for a wave, the amplitudes of each arc of the wave become gradually increased or decreased, no longer symmetric. Also, due to the experiment design in (Fig.1j), we call a wave that shapes by surface tension region to a polarized wave as a bio quantum path. (The term of bio quantum path is not only used to differing symmetric Cosine/Sine waves from surface tension polarized asymmetric waves but also means inversion superposition. In (Fig.1j), on each experimental rigid and symmetric wave arc, we have drawn some red segments, in an ideal condition, a series of these red segments compose of a quantum scale. This is the from-symmetric -to-asymmetric polarized process. We should note all superposition inside bio-systems come from polarized waves, there are no symmetric waves involved. And more generally speaking, any pattern that can demonstrate parameter-against-gravity -recovery shifting can be called bio quantum path, not just polarized waves. Life is composed of bio quantum paths.)

Suppose one series of bio quantum path $\left|\phi_{n}\right\rangle$ superpose with another bio quantum path $\left|\psi_{n}\right\rangle$, if one tide $\phi_{k}$ is damaged due to environmental impact, it is impossible for $\left|\phi_{n}\right\rangle,\left|\psi_{n}\right\rangle$, or their superposed part to repair $\phi_{k}$ if the superposition takes the conventional quantum mechanics probability. However, with bio quantum inversion superposition, the damaged $\phi_{k}$ can get Le Châtelier compensation for correction. (A musician writes accompaniment (inversion of notes) to melody is to increase the stability or driving forces of the sheet music. Equivalently, bio-systems superpose inversion structures is to increase the gravitational binding or stability of the system.) In human beings, all the wound healings, bone fracture recoveries, and even tumor cell cleanings, etc., are physical recovery processes, and the mechanism of which is the same. (We can easily understand any part that is capable of healing takes inversion superposition; and some of the parts, such as hairs, nails, horny layers, etc., no inversion superposition. Therefore, wounds induce pain, and cutting hairs, etc., will never induce pain since they don't correlate with other inversion superposition parts.)

We refer to the modified law to Newtonian 3rd Law of Motion since the original rigid body modulation equivalent action and reaction, which must under the conservation of momentum and energy. However, conventional energy is greatly non-conservative for bio-systems. Only for inversion types of energy, bio-systems are roughly conservative. (Similar to Newton's "equivalent" action and reaction, the modified law just conserves inversion energy in bio-systems; we then state that this law is modified from Newtonian 3rd law.)

(Fig. 2k(3) refers to non-living being surface tension inversion superposition, from it with the increase of gravitational binding; we can get the fundamental life unit as in (Fig. 2l(1)). This unit can be a cell, or a virus, a DNA/RNA segment, or even the primitive forms of life with surface tension inversion which we still don't know. No matter any kind of life forms including the most advanced human beings are unexceptionally inversely superposed from this simple unit. Inside the unit, we use a musical scale to represent the highest superposed bio quantum path (not necessarily the DNA or RNA) since only music can modulate inversion superposition; on the surface tension region or the outside layer, we draw some inversion units (These inversion (energy) units are evolved from diverse "reflective zones" in (Fig. 
2k(3), in advanced species are complicated; however, still originates from the basic types.). If this whole unit is a rigid body, following the Newtonian $3^{\text {rd }}$ Law the net forces acting on it will exist in equal magnitude and opposite direction. Now it becomes a life unit, then all the inversion superposition must resonate between the center core bio quantum paths and the outside surface tension layer inversion units, or the inversion energy of the two parts tend to conservative the negentropy. (We can simply regard the core bio quantum path as Planck region and the outside layer as surface tension region, then all bio-structures are inversion superposed from them.)

The simplest sheet music is at least composed of a melody and an accompaniment, all with the same scale. We can simply put the melody into the core part and the accompaniment into the surface tension region inversion part of (Fig. 2l(1)) albeit both musicians and scientists never realize that life is so naive in a pattern. No matter how simple or how complex a bio-system is. All biological functions are following this inversion resonation model. (Fig.2g), a soap bubble, and (Fig.2h), photoelectric effect, only possess surface tension inversion; therefore, just non-living beings. If a cell only has DNA and some inner cell contents but lacks the outside surface tension layer or cell membrane with enough inversion proteins, still can't be a life; real-life must function via the resonation between these two parts. Evolution takes geological time only to establish the inversion relationship between these two parts, this is what the Basic Law of Evolution physically means. Maxwell's demon is a thought experiment that tries to hypothetically explain the violation of the second law of thermodynamics, which was proposed by James Clerk Maxwell in 1867. He imagined one container divided into two parts and each filled with the same gas at equal temperatures placed next to each other and separated by a wall. An imaginary demon controls the trapdoor in the wall to only allow faster-than-average molecules, in such a way the result will be contrary to the second law of thermodynamics. As we can see, the thought experiment still only refers to the surface tension regions in (Fig. 2l(1)) (Maxwell's trapdoor wall as the surface tension region), and never refers to the resonation with the core bio quantum path; therefore, indeed cannot exist in a natural environment. However, under the conditions that satisfy the Basic Law of Evolution or in (Fig. 2l(1), we can resurrection Maxwell's demon by adding the (superposed) core bio quantum path. (Note, once Maxwell's demon is resurrected, Laplace's demon will die since people can't create a world without such surface tension "reflected" or "inversed" superpositions. With the resurrection of Maxwell's demon and the death of Laplace's demon, equivalently add inner core bio quantum path to Einstein's field equation, then bio-system gravitational binding increases for Newtonian $\mathrm{G}$ to $10^{9}$ levels due to the resonation from the core bio quantum path. We can even say, this $10^{9}$ is the "weight" of memory or negentropy. Once death, it will disappear.)

The Second Law of Thermodynamics says: $\triangle S \geq 0$, if all processes in an isolated system are reversible, the entropy is constant, otherwise, entropy generation. Also, Schrödinger had utilized his famous "cat" to question the Copenhagen story and published the term "negentropy" in his book. The Schrödinger cat plus negentropy made him as close to life as just one surface tension's thickness. However, both Clausius and Schrödinger never realized that music harmonics as a discipline centuries ago has solved the concept of the reversible process quite well. We use the simplest octave scale and triad to demonstrate a bio-reversible process in music. A scale (major) is $\left|\sqrt[12]{2^{\mathrm{n}-1}}\right\rangle$ or unfold as $\mathrm{C} \sqrt[12]{2^{0}}, \mathrm{D} \sqrt[12]{2^{2}}$, $\mathrm{E} \sqrt[12]{2^{4}}, \mathrm{~F}^{12} \sqrt{2^{5}}, \mathrm{G} \sqrt[12]{2^{7}}, \mathrm{~A} \sqrt[12]{2^{9}}, \mathrm{~B} \sqrt[12]{2^{11}}$; a triad just three major notes $\mathrm{C} \sqrt[12]{2^{0}}, \mathrm{E} \sqrt[12]{2^{4}}, \mathrm{G} \sqrt[12]{2^{7}}$ pick up from the scale with diverse inversions (must with the same "converging" position). One melody movement, accompanied by the inversion combination(s) of triads or other chords, then becomes a (bio) reversible process. No matter naive music composed by a kindergarten student, or the sophisticated music composed by Beethoven, all follow the same mechanism. If we tell Carnot or Clausius, any process with the above mechanism is a reversible process and forget about $\triangle S$ $\geq 0$. They will possibly don't believe in it. If we tell Schrödinger this is negentropy which is acquired by adding a surface tension region to his cat, a ground state can be reduced by a simple inversion superposition to attract inversion energy from a surface tension region, he will probably also not accept. However, this is what really happened in bio evolution. Thermodynamic $\Delta S=0$ reversible cycles are defined as a T-symmetry or time-reversal symmetry at least under acceptable accuracy. All those that do not satisfy this standard are regarded as "entropy generation" processes and imply as "not conducive" to their ideal purpose such as the "mechanical equivalent of heat". However, a piece of sheet music never needs T-symmetry to become "conducive". Equivalently, bio-systems never need such a T-symmetry process judgment and only need adaptation or "recovery rate". For this standard, music harmonics supplies us great tools albeit musicians never realized the melody/accompaniment relationships they used for centuries hidden the maximized system recovery rates for a series of cyclical processes based on a certain non-isolated environment. (As mentioned, Einstein's field equation only possesses an outside gravitational "shell" that lacks inversion, and Schrödinger's equation only holds the "core" Planck region and lack the outside surface tension region, the model of (Fig. 21(1)) just the resonating combination of them in one structure for gravitational binding (waves), it is a chimera of Einstein and Schrödinger but has been working well in music for centuries and in bio-systems for geological periods. We also can understand bio-reversible from some concrete examples. Every day we can hear diverse sounds for various reasons; however, people prefer to hear good music, since musical sounds are more bio-reversible patterns than non-musical sounds. Human bodies gradually trim various non-musical sounds into bio-reversible sounds by multi-surface tension regions; therefore, the closer of a structure to CSF, the closer of the oscillation patterns to Chu's constant. Not only sounds, all other parameters such as foods, water, color, gene expression, RNA profile, etc., are all trimmed in such a way to become bio-reversible and excrete "wastes". Here "wastes" just mean anything with a lower negentropy value selected by surface tension regions. Evolution "resists" environmental available resource shifting by making all in vivo processes and structures to be bio-reversible, such a "resistance" issues biological functions and drives evolution. Le Châtelier's effect is such a kind of gravitational "resistance". We also need to carefully note, in a reproduction process what transfers to offspring is memory instead of nucleotide

\section{Volume 10 Issue 9, September 2021}


sequence; the nucleotide sequence is only the "carrier" of memory; without memory to be delivered, these sequences will be dead segments. The difference between the DNA in somatic cells and germ cells is on the surface tension or memory albeit their sequence is possibly the same. All DNA segments inside the zygote need to activate surface tension later, those parts that fail to trim will be dealt with as "wastes". Milk and yolk sac are kinds of nutrients with large surface tension, babies need them is to somatically recover the surface tension of inherited DNA. Bacterial capsules follow the same mechanism.) From the "bio-reversible process" modulation, it also easily understands why we need to sleep. CSF is the largest gravitational binding or surface tension structure that inversely connects to the different parts of the body, following certain reversible process patterns to adjust the body. Any activities will leave "memory" which CSF will adjust back to the previous rest or lower entropy state. Suppose a body activity correlates CSF from $\mathrm{T} 1$ to $\mathrm{T} 12$, then the rest means reverse direction. However, such an adjustment is not a thermodynamically reversible process that fully reverses from T12 to T1; it is a bio-reversible process that only takes the major "chords" or combinations to move back since the body can't get T-symmetry food or environmental parameters. This is a daytime rest. At night, these bio-reversible CSF adjustments need further self-adjustments to flow back all daily activities, which is sleep. (This night sleep is still not full T-symmetry of daily CSF daily activities, just utilizing life unit "minimum outside impact" equals to "maximum core bio quantum path inversion" to perform the CSF self-adjustment to a more bio-reversible state.)

Nowadays, almost all prevailing hypotheses of the origin of life like to find clues for the origin of biomaterials such as RNA, DNA, proteins, etc. We must realize that the establishment of the resonation structure in (Fig. 2l(1)) should be much earlier than the existence of these nowadays known biomaterials. All known or unknown biomaterials are the inevitable evolution products from this resonation structure; never reversely happened, such as certain biomaterials or genetic code can bear such a resonation structure. The story of the RNA world without surface tension inversion resonation is still $100 \%$ non-living beings, so do the DNA segments from it. It is absolutely surface tensions that govern biomaterial functions, not reversely happened; because even non-living beings still unexceptionally possess surface tension, just weak than the former. All the inversion rules that govern bio-systems are more general than the isolated logic that originated from Newtonian times.

\subsection{The inversion (Le Châtelier effect) between inseparable Planck regions and surface tension regions in superposition process(es) originates memory that is essential to life functioning}

As mentioned, the rigid bodies or equivalent in Newtonian Third Law satisfies conservation of momentum and energy, while a rigid body evolved into the life resonation structure, the momentum and the energy formats we usually see will no long conservation. The only relative conservative energy type that can function in bio-systems is in vivo inversion (or structural) energy, difficult to transfer to any other types of in vitro energies without surface tension region. Life is a multi-surface tension structure (roughly one inversion one surface tension region). For inversion energy or structural energy, we have referred to it to some degree before. As discussed in conventional quantum mechanics, if an electron shifting between energy levels, it will emission energy. This type of energy is not inversion energy since it can transfer to diverse types but challenge inverse a structure. However, in bio-systems, if spinal inversion energy shifts from one vertebra to another vertebra, it can only accompany by inversion processes of relevant body structures since this vertebra shift includes the inversion energy of relevant body structures. (Inversion energy is inseparable from surface tension. Under in vitro conditions, due to inversion energy is so less in quantity, it is not structural energy. However, under in vivo conditions, due to almost all in vivo energy is inversion energy, then "inversion energy" becomes equivalent to "structural energy" which connects to a certain surface tension region. In conventional physics or even the whole scientific kingdom, energy is a theoretical concept which doesn't concern with structures. From Newtonian equations till Einstein's equation $\mathrm{E}=\mathrm{mc}^{2}$, energy only concerns with diverse parameters such as mass, velocity, temperature, etc., never concerns with surface tension or the structure of matter; a certain amount of mass with certain speed then gives out a certain amount of energy, no matter this mass includes high surface tension or lower surface tension. This concept is not complete, all disciplines never discriminate types of energy that can drive surface tension motions and common energy that can't accumulate by surface tension. So-called inversion energy is indeed surface tension energy or memory.)

Conventionally, the manner that people apply the law of conservation of energy is actually by means of "neglected" the surface tension inversion energy. In quantum mechanics, energy is expressed using the Hamiltonian operator. On a time scale, the uncertainty in the energy satisfies:

$$
\Delta E \Delta t \geq \frac{\hbar}{2}
$$

Eq.3 is similar in form to the Heisenberg Uncertainty Principle (but not really mathematically equivalent thereto, since $\mathrm{H}$ and $\mathrm{t}$ are not dynamically conjugate variables). Here the uncertainty item $\Delta E \Delta t$ could be regarded as a part of inversion energy. It is only a very small portion of total energy. The conventional quantum mechanics usually call its energy as "quantum (energy) level" that follows $\left|E_{n}\right\rangle=n h v$, in bio-systems we call the energy that satisfies $\left|E_{n}\right\rangle=$ $f_{0} \times \sqrt[12]{2^{\mathrm{n}-1}}$ as a "quantum scale", which can modulate more inversion energy by the combination of the scale oscillations. (The difference between $n h v$ and $\sqrt[12]{2^{n-1}}$ is the weight of surface tension region; $\sqrt[12]{2^{\mathrm{n}-1}}$ can module ideal multi-surface tension region superposition by the combinations of diverse $\sqrt[12]{2^{n-1}}$, such inversion superposition of $\left|\sqrt[12]{2^{\mathrm{n}-1}}\right\rangle$ against a system get the model in (Fig. 2l(1)). $\left|E_{n}\right\rangle=n h v$ only based on 5 postulates of conventional quantum mechanics to justify the probability, quite challenging to modulate shifting of Planck regions driven by surface tension regions. In physics, a quantum (plural quanta) is the minimum amount of any physical entity (physical property) involved in an interaction. (The fundamental notion that a physical property can be "quantized" is referred to as "the hypothesis of quantization".) From $n h v$ to $\sqrt[12]{2^{\mathrm{n}-1}}$, the "minimum physical property" notion changes fundamentally; it becomes the inversion energy. Scale is not "minimum

\section{Volume 10 Issue 9, September 2021}


physical property", it is the inversion optimization of all "parameter- against- gravity- recovery" in a system to establish reversible processes. The interaction like musical melody VS accompaniment, the ideal efficiency can be expressed in the resonation of (Fig.2l(1)). Under this ideal condition, the surface tension inversion units and core bio quantum path(s) use the same quantum scale to compose reversible processes we mentioned before. Under such environmental conditions, we believe that the inversion energy needs to transfer between the surface tension region and core bio quantum path(s) structure is zero since they compose reversible processes, or the unbalanced energy can be directly compensated from the environment.

Historically, matter and consciousness are different terms, conservation of mass is quite general in physics and even the whole scientific kingdoms; however, no available scientific model for consciousness. Now, from (Fig.2l(1)), we could realize that matter and consciousness are inseparable even from the very beginning, the consciousness has broken the conservation law since then. For matter, it will cost energy to transfers between the surface tension inversion units and core bio quantum path(s) no matter more or less; however, for consciousness, we believe that the transferring between these two special distancing structures will have no energy cost base on the system aether. For a life unit in (Fig.2l(1)), the more matter inside, the more conventional energy needs to move them. However, the more consciousness in the life unit, the more discrepancy will be presented while we use the conventional law of conservation or equivalent. We can simply say that in the real world, matter follows energy with 1unit +1 unit $\leq 2$ units and consciousness follows energy with 1 unit +1 unit $\geq 2$ units. The law of energy conservation for matter only tries to increase to close to $1+1=2$, and for consciousness works in a reverse tendency, tries to decrease to close to $1+1=2$. While we study the matter property, we can roughly use the conventional conservation law. While refers to the spiritual world or a mixture system, we should realize its energy reverse tendency.

The difference between common energy and inversion energy in bio-systems is this energy format possesses surface tension memory characteristics. (Fig.2l(2)) demonstrates a general human memory model, no matter how complex the memory processes, it still comes from the basic life unit in (Fig.2l(1) or its original version (Fig. 2k(3)). In conventional quantum mechanics, time evolution is unitary as disciplinary postulated. However, memory is non-unitary. As in (Fig.2k(3), there are some reflective zones (different size and strength) on the surface tension region, once two or move gravitational waves share the same reflective zones or inversion units for inversion superposition, then establish the memory for these gravitational waves. If more reflective zones share the same bio quantum path superposition, then establishes thinking. Memory and thinking are only characteristics of a surface tension region while it functions, here we don't differ them and all call memory. (This is a very general term across the biological kingdom that includes the origin, not the memory we usually see.) Now that it is gravitational waves that share one reflective zone and then superposition, the time is no longer unitary evolution. (In bio-systems, all superposition will induce non-unitary or inversion time. Conventional quantum mechanics takes unitary time evolution is only an approximation came from this discipline has already given up the impact of surface tension regions in quantum superposition. It is due to this reason; time in bio-systems is inversion time which concerns the in vivo gravitational structures.)

(Fig.2l(1)) is the equivalent of (Fig. 2k3), only different in superposition complexity. All life structures and even biomaterials, such a DNA segment, unexceptionally follow (Fig.2l(1) as the fundamental memory model. While evolution hits human beings, the memory model is still the same: non-unitary surface tension-driven inversion superposition to share the same reflective or inversion region. As the right hand of (Fig.2l(2), the human spinal cord composes of C1-7 cervical, T1-12 thoracic, and L1-5 lumbar spines. In the left hand of (Fig.2l(2), suppose a memory process composes of $\mathrm{C} 1, \mathrm{~T} 8, \mathrm{~L} 2$, then the hippocampus equivalent to the core bio quantum path and the cerebral cortex equivalent to the surface tension region as in the (Fig.21(1)) to compose the memory of $|\mathrm{C} 1, \mathrm{~T} 8, \mathrm{~L} 2\rangle ; \mathrm{C} 1$, T8, L2 compose of certain regions on the hippocampus, and also certain regions on the cerebral cortex, we use some arrows to connect these regions. These regions on the hippocampus connect to certain regions on the spinal cord, then connect to certain regions on the somatic body. Totally, the spinal cord composes the inversion of the brain, and the somatic body composes the inversion of the spinal cord. Each inversion is also possibly composed of a lot of sub-inversions. No matter how complex or how simple of a memory process, all fit into the inversion superposition of a simple life unit. (In advanced animal species, there are complex central nervous systems; however, they only transfer surface tension energy and increase the stability of the model in (Fig.2l), we still can use the same modulation and neglect their complexity if we don't directly study the nerves. As mentioned in an early discussion about inertia, Newtonian inertia is the intrinsic capability of an object to keep its motion state. Bio-inertia is for bio-systems to keep alive by internal motion gravitational binding in a non-isolated environment is also the intrinsic capability of surviving or issuing bio- reversible processes or structures. Bio-inertia always complies with (Fig.2l(1), if the resonation of (Fig.2l(1) reduces to a certain threshold, life will end.)

In conventional thermodynamics, the second law believes in the entropy generation by supposing an isolated system model. However, the memory model in (Fig.2l(1),(2) can be regarded as a reverse process of entropy generation. As mentioned, two or more bio quantum paths share the same reflective or inversion region on the surface tension region then composed of the memory. If we regard the two or more bio quantum paths' superposition as entropy generation processes, the reflective or inversion units used for these processes can get memory to reduce the entropy generation processes of later stage superposition, such an impact follows Le Châtelier for within-surface-tension parameters, it can become an entropy decrement process compensated by concerning surface tension region under the environmental negentropy for certain conditions. Life functions, no matter how complex or how simple, all need such kinds of memories for maintaining negentropy state activities. (E.g. among the biomaterials for a certain process, such as a piece of DNA segments, RNA, cells, etc., all have activity differences, an

\section{Volume 10 Issue 9, September 2021}




\section{International Journal of Science and Research (IJSR) \\ ISSN: 2319-7064 \\ SJIF (2020): 7.803}

active segment or an active cell are those that possess more memory or inversion energy for this process. And these memory-based activities can pass generations and be fixed in evolution. (The problem of conventional thermodynamics has still lacked the memory model. As in (Fig.2k(3), no matter gravitational binding is $100 \%$ reflected back, or in (Fig.2l(1) which is partially reflected back and partially inversion to outside, once the reflected back gravitational waves can superpose again, it will definitely induce later process entropy to reduce albeit such decrement only happens in part of the system instead of the whole system.)

\subsection{Inversion time and bio-system reversible processes}

The third law of thermodynamics bases on entropy generation belief of the second law to the absolute zero suggested the concept of the arrow of time. The unitary time evolution in quantum mechanics also stealthily relies on the "arrow of time". However, from (Fig.2l, 3a) about the modulation of memory, a memory process follows non-unitary time based on the issuing surface tension or in vivo environment, and the level of non-unitary capability corresponds to evolution levels. E.g. a human knows much more than a lower evolved animal can be regarded as the surface tension of the human cerebral cortex can inversion much wide time range than the cerebral cortex of an animal, or it contains more inversion time. The time in bio-systems is never showing a single direction "arrow" as claimed even if the inversion of time in bio-systems is not always symmetric. A surface tension region can make time into non-unitary while it reflects (or inverses) gravitational waves from the very beginning. The time of a wave and its surface tension reflecting counterpart, or two reflected gravitational waves at different stages can't be unitary. Such non-unitary property is where negentropy originated from, memory happens in the reverse direction of entropy generation, steering each species in the whole biological kingdom to functioning with bio-reversible structures and processes. Food digestion is a process that makes use of the non-unitary characteristics of surface tension to acquire negentropy; gut peristalsis is also to get more negentropy, from the process, only the lower entropy part of the same ingredient can be absorbed into the gut. (The reason why there are many valves and folding structures on the intestine is also to acquire inversion time simultaneously.)

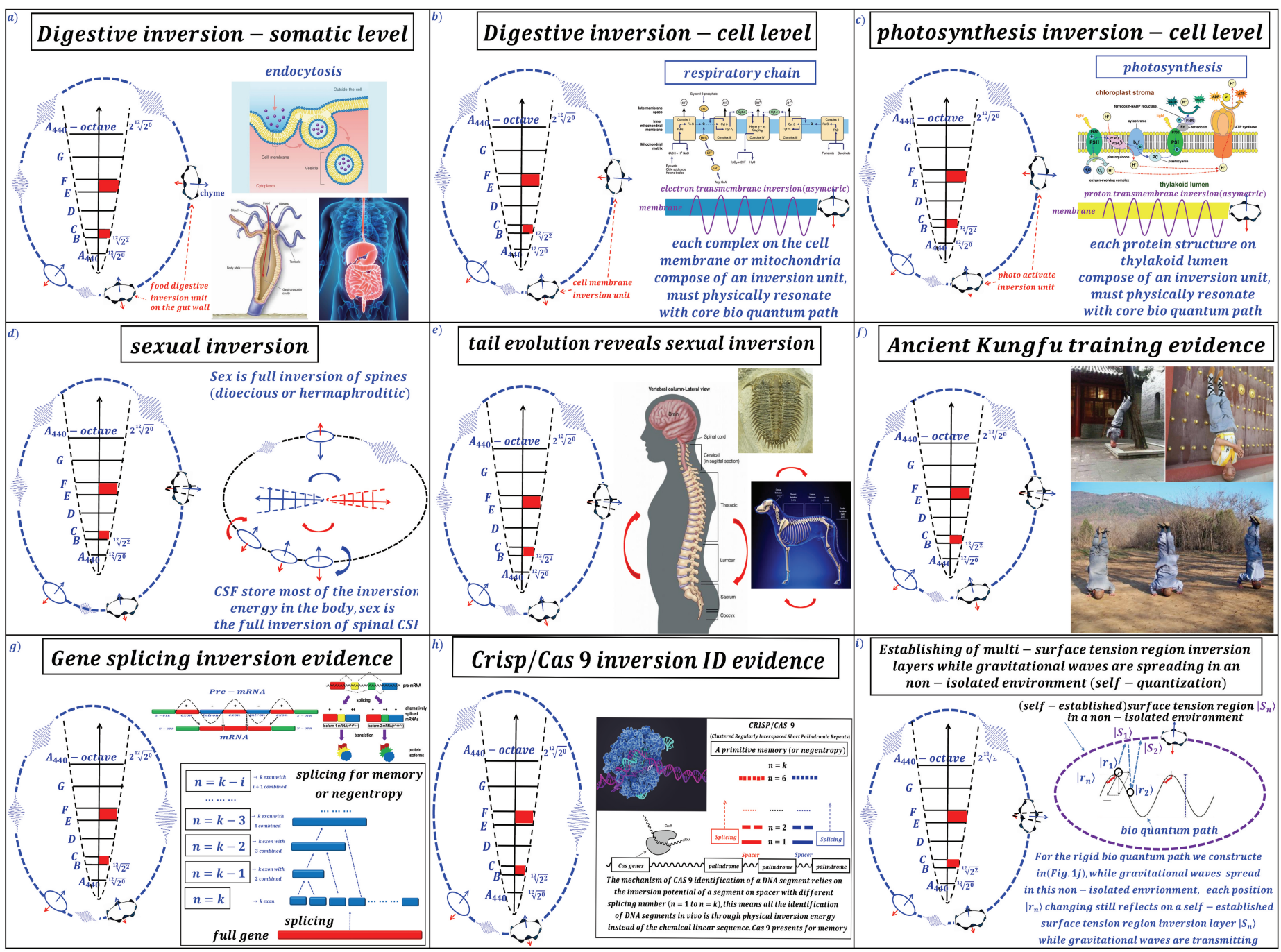

Figure 3: Memory characteristics of inversion energy and its surface tension driving functions

a) digestive inversion at the somatic level b) digestive inversion at the cell level c) photosynthesis inversion at the cell level $\mathbf{d}$ ) sexual inversion model e) tail evolution reflect the spinal inversion f) ancient kungfu training system evidence for spinal inversion g) modern gene splicing inversion for negentropy model h) CRISP/CAS 9 inversion model for gene identification and genomic immunity i) a model for the tendency of self-establish of surface tension region in a non-isolated environment while gravitational waves are transmission 
(Fig.3a) demonstrates the food digestive inversion which takes the same modulation of (Fig.2l(1)) at the somatic level. Left of (Fig.3a) shows the evolution of the digestive systems from single-cell endocytosis, to hydra, and to humans. For endocytosis, the vesicle is established by inversion of the cell membrane; therefore, even a vesicle is inside the cell, the physical exchange between the vesicle wall and in vivo cell environment should be equivalent to the surface tension inversion units on the left hand of (Fig.3a). Similarly, from the trans-somatic body digestive canals from hydra till humans, all foods inside them are still physically regarded as surface tension inversion, digestive canal wall become the inversion units of the life unit on the left hand of (Fig.3a). No matter which evolution level of food digestion, all can be fit into this model. For humans, the food ingredients are repetitively in-and-out of the gut wall compose of the outside surface tension inversion layer oscillation, and spinal cord CSF oscillation composes of core bio quantum path inversion. The oscillation of these two parts following the basic law of evolution composes of the digestion and excrement process, the higher inversion parts can be modulated by Chu's constant at the somatic level. (Suppose there is an amino acid in vivo inside the blood and there is the same amino acid in the gut chyme, the entropy of these two amino acids is quite different. The amino acid inside the gut must be inversion in-and-out of the gut wall for many rounds and then can get the same entropy with the same amino acid in the blood or they belong to the same surface tension region. Only after this internal motion adaptation process, the amino acid can then be absorbed by Le Châtelier's effect. The amino acid example is only locally happening. The in-and-out processes still need to be further adjusted through a holistic surface tension region to be bio-reversible. The small intestine itself is a large surface tension region, digested intestinal juice surface lining the small intestinal walls. While part of the nutrients is finally absorbed into a certain location of the intestinal walls, the whole intestinal surface continuously peristalsis to make the high surface tension absorption active ingredients tend to a bio-reversible pattern. The digestive capability difference between an old man and a young man is only the inversion number; for a young man, the food ingredients can repetitively inversion in-and-out of the gut wall for many rounds, with the degree of senescence, such an inversion number is gradually decaying.) The digestive mechanism of other bio-systems including lower evolved single-celled species, all follow this bio-reversible process model. From here we can understand that digestion is still driving by memory, or it takes the same physical model. The "memory" we usually know is only a small fraction of the whole memory of an organism.

(Fig.3b) shows the digestive inversion at the cell. A series of respiratory chain complexes on the cell membrane or mitochondrial cristae compose of the surface tension inversion. Electrons cross each membrane complex from reducing power (NADH, NADPH, etc.) to electron acceptors $\left(\mathrm{O}_{2}, \mathrm{NO}_{3}^{-}, \mathrm{SO}_{3}^{2-}, \mathrm{Fe}^{2-}\right.$, etc. ), following the basic law of evolution, such a cross membrane inversion should resonate with the inner bio quantum path superposition, Mitochondrial DNA possibly plays the role. (Convention quantum mechanics can only modulate electrons of elements, can never modulate such kind of cross membrane electron behavior due to lack of inversion.)
(Fig.3c) shows the photosynthesis at the cell level, a process reversed in the direction from respiratory in which electrons raise electromotive potential from electron acceptors to reducing power, or call proton transmembrane inversion. This process still follows the life unit resonation.

Memory can let organisms acquire negentropy for life function; however, it can't make life generation becomes eternal. As in the memory model, a series of bio quantum paths superposition $\left|\Psi_{n}\right\rangle$ composed of an event and leaves memory $\left|k_{n}\right\rangle$ on the surface tension region. Later, another series of bio quantum path superposition $\left|\phi_{n}\right\rangle$ events in which the inversion number will slightly impact by the memory $\left|k_{n}\right\rangle$, or potion of the inversion energy has been transferred from $\left|\Psi_{n}\right\rangle$ to $\left|\phi_{n}\right\rangle$ albeit their time is non-unitary. As mentioned, the inversion number on the same surface tension represents the level of negentropy, bio-systems can then use this way via surface tension region to acquire negentropy. With more and more memory events happen on the surface tension, inversion time will use out or memory function will be dampened, then life will be ended. As the basis of Life, surface tension is not eternal, then the lifespan for generation is limited.

However, limited generation lifespan never stops evolution moving forward by cross-generation negentropy transmission. In conventional quantum mechanics, the ground state of a series of energy levels can't be reduced since it fully excludes surface tension. As mentioned in the basic law, under the same surface tension or in vivo environment, the structures with more inversion rounds will possess lower entropy or more gravitational binding than those with less inversion round number; therefore, one series of scale $\left|\sqrt[12]{2^{n-1}}\right\rangle \uparrow$ superposed with its full inversion $\left|\sqrt[12]{2^{n-1}}\right\rangle \downarrow$ will get a lower than parent entropy ground state via surface tension since this process superpose two surface tension potential together. If this lower entropy ground state finally gets developed, the result will be a full lower entropy scale. The rate of the lower ground state get full development is not high. However, this process is impacted by parent generation memory, never a random variation. (All the randomly happened nucleotide sequence shifting will still be made into a bio-reversible pattern to resist the change. Even the mutation signal is random, the selection and fixation process are no longer random. For the reproduction process, the meiosis and mitosis modulation in conventional genetics possibly also need modification, the DNA sequences from parents are less likely $50 \%$ ratio. The oval cleavage process is a standard memory process to adjust the ratio.) This is the cross-generation negentropy transmission in biological reproduction and evolution. (Fig.3d) shows a general sex inversion model for bio-systems; in human beings, it means the full inversion of the spinal cord CSF. From invertebrates to single-celled lower-evolved species, the mechanism of acquiring sexual negentropy is the same, just using full inversion of the largest gravitational binding structure. (A man and a woman can fall in love only because their spinal cords oscillate with a close reversible process scale. Therefore, love is still the special transmission of in vivo gravitational binding in a short range, never escape the steering of surface tension regions.) 
The negentropy induced by full inversion of the largest gravitational binding only presents on the ground state location or nearby structures never happens in the whole system. In human or animal sexual behavior, sperms become the lowest entropy part that goes out of the in vivo environment, for this reason, the entropy of parents will be unavoidably decreased and wait for later recovery. The process of reducing the ground state or acquiring negentropy generally will make the lower ground state part automatically detach from the previous structures, we can call it "ground state-generation-driving forces" which still originate from surface tension. The driving force of enough surface tension nutrients absorption into cells, or animal sperm ejaculation, etc., is the same sort of "ground state-generation-driving force" across the whole biological kingdom. And in each ejaculation, there are a lot of spermatozoa; finally, only the spermatozoon with the same surface tension oscillation frequency with that of the oval can get insemination. Following the life unit in (Fig. 21(1)), the inversion on the sperm surface and the oval surface physically oscillates with their inner genomes to compose certain bio-reversible processes; therefore, before insemination, the genome of the sperm and oval make a physical bio-reversible process selection again. Both the oval and the sperm full genomes get a reversible process inversion on their surfaces which is reversible with their inner genomes, the final successful sperm should be those with a surface tension inversion frequency close to those from the oval. In conventional quantum mechanics, the ground state only uses to justify the correlation between measurement and quantum state; also, never concerns with entropy and gravitational binding. However, in bio-systems, surface tension binds all these concepts together. (For sexual loss of gravitational binding or negentropy, experimental validation is relatively easy as our previous (Tab. 8), just it is a little challenging to test the gravitational binding after digestion of foods by $F D H s$.)

Animal and human tail evolution offers some indirect evidence for sexual inversion. In early lower evolved species, the distance between the brain and the sex organ is small (such as a Trilobita). With the progress of evolution, this distance is gradually enlarged. Such an enlargement means the accumulation of surface tension. Till vertebrates as in the right hand of (Fig.3e), sexual organ roughly locates in the middle of the spinal cord and the tail. Therefore, in sexual behavior, the spinal cord and the tail establish the sex inversion that generates negentropy for the offspring. (The CSF in the vertebrae correlates with a different part of the body. Sexual behavior drives the CSF in the spinal cord section to the tail location and the CSF in the tail mainly goes out with the sperms, and the inversion of this highest gravitational binding structure offer sperms with much negentropy.) While evolution hit human beings, the somatic surface tension increased greatly, the tail no longer needs to participate in the sexual inversion, therefore, no CSF inside the caudal vertebrae. For humans, there are 5 lumbar vertebrae, generally the second to the fourth lumbar vertebrae function as the sexual inversion center for different people. That means in sexual behavior the CSF above this center is inversed with the CSF below this center and accompanied with relevant somatic change.
The above models about memory, food digestion, and sexual behavior had been applied in ancient Chinese Kungfu for a long time. Shaolin, with more than 1500 years of history, and Wudang, with 590 years of history, have systematic physical training methods incorporated albeit ancient people lacked theoretical modulation and modern knowledge for the explanation. (Fig.3f) is one of the easy understanding training methods for somatic inversion. Starts at around seven years old with a headstand till very old ages, this training is a kind of basic spinal inversion training at the somatic level. Spinal CSF is the highest surface tension or gravitational binding structure in human bodies, for most people's daily activities the head is always higher than the feet, this training force the body to adapt to a reverse direction CSF; therefore, increases the surface tension of CSF or makes the CSF coordinate well with the somatic body. This training can increase the sexual performance of people; however, like most of the ancient kungfu training methods which directly targeted CSF, must take abstinence way of living style to increase lifespan. Shaolin totally forbids any sexual behavior or related. Wudang, albeit not fully forbids, has sexual control kungfu system in which sexual behavior is only technologically arranged for reproduction and never for pleasure like modern society. Since sexual behavior refers to the full inversion of the spinal CSF, if any physical training or aphrodisiac drug can increase the sexual performance of a person, they will unavoidably impact the recovery capability of the spine or the lifespan if not arranged in a controlled manner.

Hypertension and cerebral hemorrhage are modern problems dangerous to people over 40 years of age. Modern medical sciences never regard it as a physical disease and generally offer chemical medications which side effects are challenging to control. The training in (Fig.3f), if started earlier, even use individually, can significantly solve the modern problems of hypertension and cerebral hemorrhage at an old age. If this training only hopes to reduce hypertension at old age and not for training Kungfu or to increase sexual capability, then no need to consider sex forbidden.

Different from the modern Olympic Games which originated from Ancient Greece, Rome and emphasize muscle strength and various techniques but rarely train spinal cord; ancient Chinese kungfu took another way, people must integrate spinal physical training as the basis for muscle and any other training. We have referred to Wudang converging and Shaolin converging training before $\left((\mathrm{p} 1707, \rightarrow)^{1}\right.$. Also, (Suppl.Movie 11) shows a preparation training as well as lifetime training. The purpose of this training is to increase the surface tension gravitational binding of each vertebra as well as the whole CSF. (Like all ancient training that refers to CSF, if not control sexual behavior, will decrease lifespan instead of benefits health.) All these have offered evidence for the basic law.

Besides ancient systems, modern biological sciences still "unintentionally" offer board evidence for the basic law. As in the top right of (Fig.2g), all genes in eucaryotes need "RNA splicing" which spins off introns (3'AG-----GU-5') from pre-mRNA to reconstruct into mature. Intron to intergenic sequence is around 1:1 across animal species ${ }^{15}$ and a large gene will then cover a lot of palindromes in scale. Alternative splicing ${ }^{16}$ is claimed to result in large numbers of protein 
isomers and for humans, 95\% of multi-exonic genes are alternatively spliced ${ }^{17}$. It is usually claimed that the purpose of alternative splicing is to "increase isomer number for adaptation" and Drosophila Dscam which potentially produces 38,000 isomers $^{18}$, possibly with the highest hypothesis isomer number in literature. However, we believe that the purpose of splicing is for the negentropy or memory of the segments; among a large number of combinations, only a small part of them acquire enough memory and go to the next step, for those segments fail to get enough memory will go to decomposing process. As in the bottom right of (Fig.2g), the surface tension of that environment first issue exons base on previous "memory", we suppose that in vivo surface tension can give $\mathrm{K}$ number of exons. For these $\mathrm{k}$ number of exons, they potentially have different kinds of combinations, such as $\mathrm{k}-1, \mathrm{k}-2, \ldots . . \mathrm{K}-\mathrm{i}$ as in the figure. However, any combination must be acquired by inversion superposition that is also driving by that on-site in vivo surface tension. DNA is inside a nuclear membrane which memory regulates DNA mobility. RNA splicing goes out of the nuclear membrane thus the memory comes out from the relevant cell membrane or endoplasmic reticulum inversion. Follow the basic law, the more inversion number of the acquired exon combinations, the more negentropy of the protein isomers. Among so many splicing and reconstruction exons, only those finally get enough surface tension or memory can be activated and go to the next step base on the on-site "memory", or we can say that the successful candidate acquires negentropy by sacrifice a large number of other combinations under the same surface tension (or inversion units). Such a "sacrifice" process is the same mechanism as digestion or sexual ejaculation we mentioned before. (Under in vitro conditions, no matter how many rounds a cyclic process runs, we believe the gravity of a system is not changed. However, under in vivo conditions, surface tension greatly enlarges internal motion gravitational binding. We can then roughly use the cyclic process round number directly to represent gravitational binding or negentropy even if we don't know the exact scale, the relevant inversion units on the surface tension region of the process, or which ingredients are inside the cycle. The inversion round number for all processes is self-adjusted by the in vivo environment to a bio-reversible condition. All surface tension memories can be regarded as evolved to supply the bio-reversible process for a certain process; a cyclic goes in one way, then surface tension will supply it with inversion bio-reversible process-based adaption. From here, we can also understand the difference between conventional quantum mechanics superposition and bio-system inversion superposition. In quantum mechanics, "observation" makes the wave function collapse to a single eigenstate, and it still doesn't know the wave function recovery condition. In bio-systems like the RNA splicing example, RNA segment can be regarded eigen-sequence, memory can be regarded as the quantum state. The interaction between these two parts driving the splicing process. Once surface tension exists, there will no such thing as "collapse" since the whole surface tension finally connects to CSF which can compensate for the cell splicing to a large degree; and all the in vivo functions are activating on a condition which conventional quantum mechanics believes certain wave functions will collapse. If the memory of a certain segment can't be finally recovered, then it will be decomposed.) For this model, a lot of indirect evidence is available. Recursive splicing ${ }^{19,20}$ is another type of alternative splicing, for large intron need to be spun off by multi-steps and needs days to finish. Self-splicing occurs for rare introns that form a ribozyme, performing the functions of the spliceosome by RNA alone. Studies from a wide range of organisms show that the intron-exon structure of homologous genes in different organisms can vary widely ${ }^{21}$. More recent studies of entire eukaryotic genomes have now shown that the lengths and density (introns/gene) of introns varies considerably between related species $^{22}$. Intron loss is demonstrated to correlate with gene expression ${ }^{23,34}$,etc.

In prokaryotes, there is no intron/exon. However, CRISP/Cas9 still takes the same bio-reversible memory mechanism with eukaryotic gene splicing for segment identification and system immunity. The CRISPR array comprises an AT-rich leader sequence followed by short repeats that are separated by unique spacers. CRISPR repeats typically range in size from 28 to $37 \mathrm{bp}$, though there can be as few as $23 \mathrm{bp}$ and as many as $55 \mathrm{bp}$. The size of spacers in different CRISPR arrays is typically 32 to $38 \mathrm{bp}$ (range 21 to $72 \mathrm{bp}$ ). There are usually fewer than 50 units of the repeat-spacer sequence in a CRISPR array ${ }^{25}$. As in right of (Fig.3h), we use a blue and a red-colored DNA segment in the spacers that will be splicing into segments from $n=1$ to $K$. Suppose the blue segment is a host segment, its functional translation will leave a memory on the surface tension region. While the red segment into the spacer, the memory of the blue segment will splice it into the same splicing number $(n=k)$ or bio-reversible equivalent splicing number with the blue segment, if these spin off $\mathrm{k}$ segments leave the same "memory" on that surface tension region, then the red segment will belong to host; otherwise, will be identified as the invading segments and destroy. A segment and all its bio-reversible inversions under a certain in vivo environment will be identified as the same category. We still use the same example from the previous for understanding. Suppose a segment with seven genes oscillate into a scale as $\mathrm{C} \sqrt[12]{2^{0}}$, $\mathrm{D}^{12} \sqrt{2^{2}}, \mathrm{E} \sqrt[12]{2^{4}}, \mathrm{~F}^{12} \sqrt{2^{5}}, \mathrm{G} \sqrt[12]{2^{7}}, \mathrm{~A} \sqrt[12]{2^{9}}, \mathrm{~B} \sqrt[12]{2^{11}}$; the simplest triad in major is $\mathrm{C} \sqrt[12]{2^{0}}, \mathrm{E} \sqrt[12]{2^{4}}, \mathrm{G} \sqrt[12]{2^{7}}$, then all the combinations of the genes follow the same converging pattern are belonging to host. However, if certain triad combinations change the converging position, such as $\mathrm{C} \sqrt[12]{2^{0}}$, \#D $\sqrt[12]{2^{3}}, G \sqrt[12]{2^{7}}$ (note the shifting from $\sqrt[12]{2^{4}}$ change to $\sqrt[12]{2^{3}}$ ), then they belong to invading, will go to decompose. No matter how these gene combinations changes, once the converging position is the same, they belong to the host, and once the combination converging position changes, they belong to invading. The case 9 issuing RNAs just identify the segment combinations by synthesis the same converging RNA or different converging RNA for the purposes. Bacterial transformation is a process of incorporation of exogenous genetic material from its surroundings, the incorporated genetic materials should be able to activate the memory based on the interaction between the cell membrane and environment. Bacteria can't discriminate nucleotide sequence but can discern from their own surface tension memory that certain segments can be incorporation well. (We should carefully note, Codons or equivalents are the evolution results of the interaction between linear nucleotide sequence and relevant memory, not the original "codes" that drive evolution and no species including the most advanced one can directly

\section{Volume 10 Issue 9, September 2021}


"discern" them. The "competence state" for incorporation is also due to surface tension memory.)

From here we can clearly know, no matter splicing in eukaryotes or the Crisp/Cas 9 identification in prokaryotes, all follow the surface tension "memory" (this primitive "memory" means the splicing and reconstruction numbers against the on-site condition for negentropy.) on a certain surface tension region, never follows the linear nucleotide. (Use the surface tension "memory" to identify a DNA segment will maximize the tolerance of linear nucleotide variation by adaptation. For linear sequence, one nucleotide change for various reasons will induce a great impact on all genes on the whole DNA segment. Utilizing "memory" and inversion can avoid such impact to a large degree, also potentially driving genetic favorable mutations even from invading segments. Till now, conventional genetics linear nucleotide sequence story never knows what a favorable mutation is; however, genetic duality system with nucleotides and memory will establish favorable mutations under certain conditions.) From here, it is easily understood that genes plus physical surface tension region "memory" have already composed a perfect evolution system that smoothly integrates conventional genetic copy and genetic variation concepts. For immunological sciences, immune response patterns also need certain surface tensions. The memory B cell is not the original memory concept, however, still needs the original surface tension memory as the basis, so does the more advanced human memory we usually see. (Human memory generally can't compete with a computer for accuracy since the basis of it is surface tension reflected or inversion format. However, it possesses real creativity. Such creativity is still equivalent to the mechanism of Crisp/Cas 9 integrating genetic copy and genetic mutation by surface tension memory.) The concept of innate immunity which still lacks a modulation in the discipline could be regarded as the surface tension or gravitational binding stored in CSF that nowadays only can be tested by our FHDs. (In conventional biological or medical sciences, people try to test the chemical ingredients inside CSF to find something. Some even claimed that they had invented a continuous CSF sampling system for animals. However, the primary function of CSF is the largest surface tension pool of the body, without realizing this role and only blindly focus on chemical ingredients can't solve any problem. While we disciplinarily use linear nucleotide sequence to decode genes and forget any sequence need surface tension memory, we are making the same mistakes.) We have to say that modern biological sciences should integrate surface tension memory into their disciplines, equivalently does modern physics. The shortage of surface tension memory is in the disciplinary range, not just in certain research cases.

Back to the rigid bio quantum path we constructed in (Fig.1j), we believe while the metal ball running on it, a self-established surface tension region should also exist, based on our previous studies for bio-systems. As mentioned, Einstein's gravitational waves travel at the speed of light; however, Einstein only estimated the range, never show a concrete frequency, also his field equation needs to assume a vacuum environment. Possibly in (Fig.3i) with our rigid bio quantum path under a non-isolated environment, there exist a surface tension region that comes from "parameteragainst-gravity-recovery" interacts with the environment such as air molecules, etc., then can establish a surface tension; we then drew a hypothesized surface tension region by a dotted line to enclose the rigid bio quantum path in right of (Fig.3i). The range may be rough 1-2m. Part of the gravitational waves possibly reflects or inverses back while the metal ball is running on the rigid bio quantum path. The position of the ball can be written as $\left|r_{n}\right\rangle$ and the reflective surface tension region that comes from the environment can be written as $\left|s_{n}\right\rangle$. Then each position of the metal ball such as $\left|r_{1}\right\rangle,\left|r_{2}\right\rangle, \ldots .\left|r_{k}\right\rangle$ on the bio quantum path can reflect on the hypothesized surface tension region we drawn to leave "memory" as $\left|s_{1}\right\rangle$, $\left|s_{2}\right\rangle, \ldots\left|s_{k}\right\rangle$, then the system can bind gravity. Due to all surface tension regions possess non-unitary time; this "invisible surface tension" first demonstrates the time effect as we have seen. The in vivo gravitational waves should follow nonrelativistic conditions. We believe in the process of gravitational waves traveling, it is the surface tension we drew as in (Fig.3i) that accumulates nonrelativistic gravitational binding that drives evolution.

If we abruptly draw such a surface tension for a ball running on a wave-shaped orbit and explain a nonrelativistic gravitational wave story, people will be reluctant to accept it. However, we can't find any exception in the whole biological kingdom, in our non-living being experiments, as well as board observations. If we go back to the earlier Newtonian Law $\vec{F}=G \frac{m \cdot M}{r^{2}}$, or later Poisson version: $\nabla^{2} \Phi=4 \pi G \rho$, we can easily understand that the prerequisite condition for this law is that there is no surface tension interference between $\mathrm{m}$ and $\mathrm{M}$, or $\mathrm{m}$ and $\mathrm{M}$ under the same surface tension region thus no large internal motion difference between them. Once this prerequisite condition is broken and then life evolution will be initiated; with the progress of life evolution, $10^{9}$ levels of discrepancy will fall on this law which is induced by surface tension regions. The phenomenon that gravitational waves can establish surface tension regions in the processing of spreading in a non-isolated environment can be called as gravitational "self-quantization". Evolution relies on the self-quantization of in vivo gravitational waves in such an environment.

\section{Method Section}

The method in this study involving investigate gravitational binding impacts on objects' FHDs (falling height difference) at free fall state, ranging from close to rigid body objects, liquids, till organisms including human beings. We first compared the FHD of slinky objects with internal motion and those objects without internal motion to differentiate the origin of $F H D$. Then test the FHDs of a half-bottle of oil and a half bottle of water, using liquid nitrogen treatment as CK to confirm that the FHDs come from internal motion.

To test the FHDs of organisms, we compared the dead and alive states of organisms, and also used liquid nitrogen-treated organism pairs to confirm that the FHDs of the alive state still come from internal motion. Finally, we tested the human FHDs from a $10 \mathrm{~m}$ diving platform, the FHDs of animals before and after sexual behavior, and the FHDs of plant raw seeds and germinated seeds from the same batch.

Bio quantum path experiments are used to define inversion energy. Also involves board discipline studies 
including conventional physics, biological sciences, and ancient China Kungfu training.

\section{Acknowledgements}

Thanks to the long term ancient Kungfu training in mainland China, five years of Ph.D. training in Tsinghua University medical school, Beijing, from 2011 to 2016. Thanks also to the 6-year kindergarten girl from Dorset Drive Public School in Ontario Canada offered a pair of ice-cream clips and participated in part of the experiments.

\section{Ethnical Statement}

All methods were carried out in accordance with relevant guidelines and regulations. The mammal animal intervention tests have been approved by the Tsinghua University Animal facility with (IACUC) approbation. All human volunteer participants in this study only refer to a sportive test for FHDs to physically understand gravitational binding inversion energy, without any somatic intervention and no biological tissue samples were taken out from human bodies.

\section{Authors' Contributions}

L.Y.Y. perceived the models, performed all the experiments, and wrote the manuscript.

\section{Competing Financial Interests}

The authors declare no competing financial interests.

\section{Supplementary information}

Supplementary information is available in the online version of the paper.

Supplementary movie 1 to 11 and datasets can also be downloaded at:

ScienceDB, CSTR: 31253.11.sciencedb.02448,

DOI: $10.57760 /$ sciencedb.02448,

entitled "Measuring human in vivo gravitational waves and the origin of an elastic reference memory".

Supplementary Movie 1. part of the Apollo 15 experiment video on the Moon (1971 by astronaut David Scott)

Supplementary Movie 2. the movement of a free suspension slinky after release

Supplementary Movie 3. the FHD of a metal chain and a same length slinky from $7.05 \mathrm{~m}$

Supplementary Movie 4. the FHD of a half-bottle of oil and a half bottle of water from $7.04 \mathrm{~m}$-video by a 6-year-old kindergarten girl from Dorset Public School, Brampton, Canada

Supplementary Movie 5. bio quantum path experiment for definition of bio-inertia
Supplementary Movie 6. the FHD of a dead(cooked) apple and an alive apple from 7.04m_video by a 6-year-old kindergarten girl from Dorset Public School

Supplementary Movie 7. the $F H D$ of a rat and a metal weight from $7.04 \mathrm{~m}$

Supplementary Movie 8.the $F D H$ of soybean seeds after soaking $24 \mathrm{H}$ against raw seeds from $7.04 \mathrm{~m}$

Supplementary Movie 9. accepted diving posture 1

Supplementary Movie 10. accepted diving posture 2

Supplementary Movie 11_ancient training for increasing the surface tension of spinal vertebrae

\section{References}

[1] Lai, Y. Y. Bio-inertia resonates life into evolution. IJSR, 8, 1680-1718 (2019). (*** for the reference format $(\mathrm{p} 1681, \rightarrow)^{1}, \mathrm{p}$ means page, $\rightarrow$ " $\rightarrow$ means the referred clause presents more than one time in the paper. Reference paper 1 only offers reliable historical data. However, at the publication time, some experimental methods and understanding are incomplete. Therefore, for any points of view discrepancy, please refer to the updated new paper and forget the old paper use only for the historical records.)

[2] Ciufolini, I., Wheeler, J. A., Gravitation and Inertia. Princeton, New Jersey: Princeton University Press, pp117-119(1995)

[3] [In Latin] Isaac Newton's Philosophiae Naturalis Principia Mathematica: the Third edition (1726) with variant readings, assembled and ed. by Alexandre Koyré and I Bernard Cohen with the assistance of Anne Whitman (Cambridge, MA, 1972, Harvard UP)

[4] Eötvös, L. Mathematische and naturnissenschaftliche Berichte aus Ungarn 1889; 8 65; Annalen der Physik (Leipzig) 6811 (1922). Physical Review D. 61(2): 022001(1999).

[5] Fray, S., Diez, C. A., Hänsch, T. W. \& Weitz, M. Atomic interferometer with amplitude gratings of light and its applications to atom-based tests of the equivalence principle. Phys. Rev. Lett. 93, 240404 (2004).

[6] Zhou, L. et al. Test of Equivalence Principle at $10^{-8}$ Level by a Dual-Species Double-Diffraction Raman Atom Interferometer. Phys. Rev. Lett. 115, 013004(2015). 
[7] Rosi, G. et al. Quantum test of the equivalence principle for atoms in coherent superposition of internal energy states. Nat Commun 8, 15529 (2017).

[8] Zurek, W. H. "Quantum Darwinism". Nature Physics. 5 (3), 181-188(2009).

[9] chlosshauer, M. Decoherence, the measurement problem, and interpretations of quantum mechanics. Rev. Mod. Phys. 76 (4), 1267-1305(2005).

[10] Einstein, A., Podolsky, B. and Rosen, N. Can Quantum-Mechanical Description of Physical Reality Be Considered Complete? Physical Review. 47, 777-780 (1935).

[11] Dirac, Paul A.M. (1982) [1958]. Principles of Quantum Mechanics. International Series of Monographs on Physics (4th ed.). Oxford University Press. p. 255. ISBN 978-0-19-852011-5.

[12] King, N., Hittinger, C.T., Carroll, S.B. Evolution of key cell signaling and adhesion protein families predates animal origins. Science. 301, 361-3(2003).

[13] Greiner, W. (2001). Quantum Mechanics: An Introduction. Springer. ISBN 978-3-540-67458-0.

[14] Barrett, J. E., Diane C. W., Avelina Q. P.,Gary W. C. Embryonic quail eye development in microgravity. $J$ Appl Physiol 88, 1614-1622(2000)

[15] Francis, W.R., Wörheide, G. Similar ratios of introns to intergenic sequence across animal genomes. Genome Biol. Evol. 9(6),1582-1598(2017).

[16] Black, D. L. Mechanisms of alternative pre-messenger RNA splicing. Annual Review of Biochemistry. 72 (1), 291-336(2003).

[17] Pan, Q., Shai, O., Lee, L.J., Frey, B.J., Blencowe, B.J. Deep surveying of alternative splicing complexity in the human transcriptome by high-throughput sequencing. Nature Genetics. 40, 1413-1415 (2008).

[18] Schmucker, D. et al. Drosophila Dscam is an axon guidance receptor exhibiting extraordinary molecular diversity. Cell 101, 671-684(2000).

[19] Sibley, C. R. et al. Recursive splicing in long vertebrate genes. Nature 521 (7552), 371-375(2015).

[20] Gazzoli, I. et al. Non-sequential and multi-step splicing of the dystrophin transcript. RNA Biology, 13, 290$305(2016)$

[21] Rodríguez-Trelles, F., Tarrío, R., Ayala, F.J. Origins and evolution of spliceosomal introns. Annu. Rev. Genet. 40, 47-76(2006).

[22] Mourier, T., Jeffares, D.C. Eukaryotic intron loss. Science 300, 1393-1393(2003).
[23] Jeffares, D.C., Penkett, C.J., Bähler, J. Rapidly regulated genes are intron poor. Trends in Genetics. 24, 375-8(2008).

[24] Castillo-Davis, C.I., Mekhedov, S.L., Hartl, D.L., Koonin, E.V., Kondrashov, F.A. Selection for short introns in highly expressed genes. Nature Genetics. 31, 415-8 (2002).

[25] Barrangou, R., Marraffini, L.A. CRISPR-Cas systems: Prokaryotes upgrade to adaptive immunity. Molecular Cell. 54 (2), 234-44(2014).

Volume 10 Issue 9, September 2021

www.ijsr.net

Licensed Under Creative Commons Attribution CC BY 